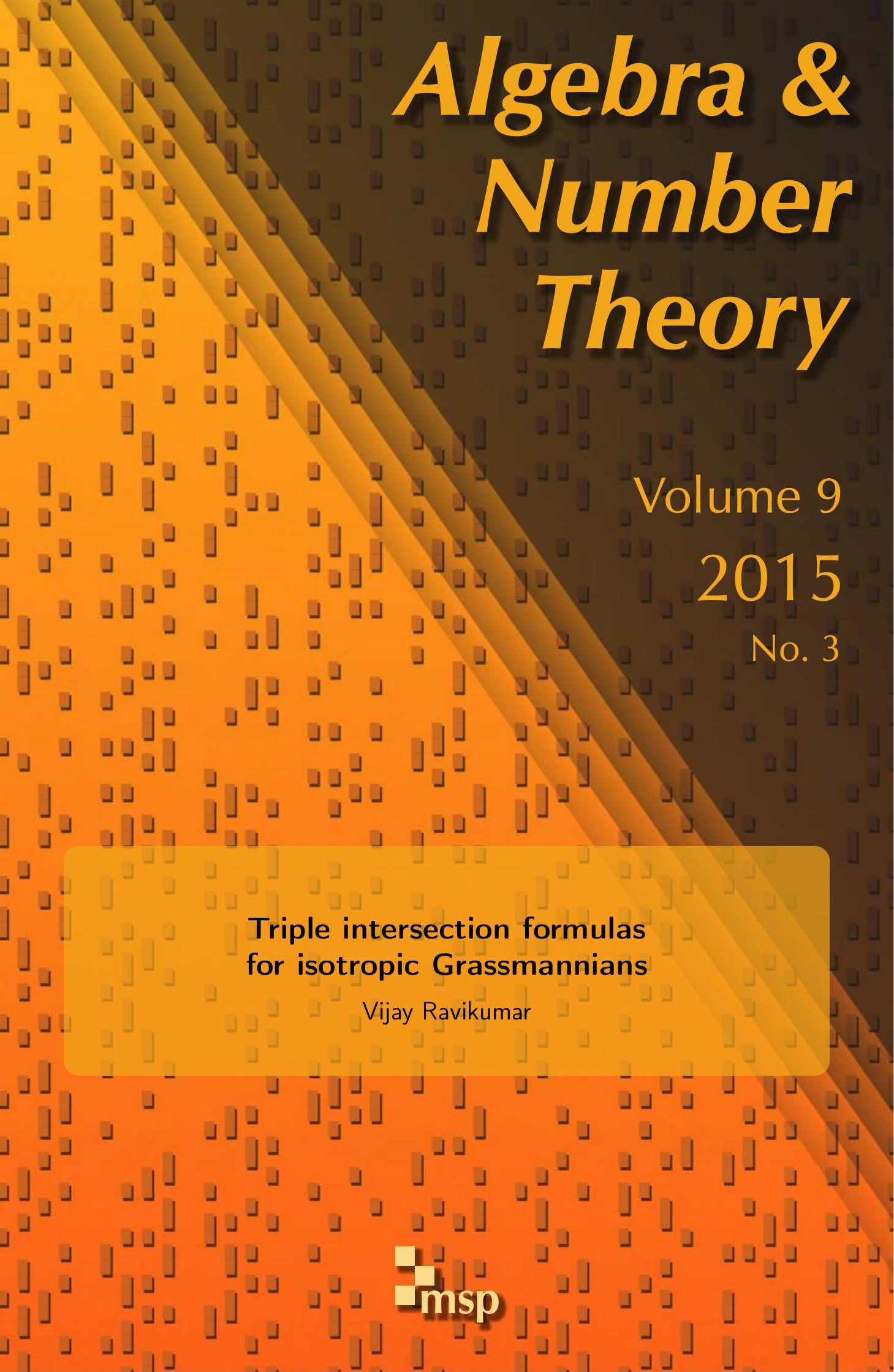




\title{
Triple intersection formulas for isotropic Grassmannians
}

\author{
Vijay Ravikumar
}

Let $X$ be an isotropic Grassmannian of type $B, C$, or $D$. In this paper we calculate $K$-theoretic Pieri-type triple intersection numbers for $X$ : that is, the sheaf Euler characteristic of the triple intersection of two arbitrary Schubert varieties and a special Schubert variety in general position. We do this by determining explicit equations for the projected Richardson variety corresponding to the two arbitrary Schubert varieties, and show that it is a complete intersection in projective space. The $K$-theoretic Pieri coefficients are alternating sums of these triple intersection numbers, and we hope they will lead to positive Pieri formulas for isotropic Grassmannians.

1. Introduction

2. Preliminaries 1: types $B$ and $C \quad 685$

3. Preliminaries 2: type $D \quad 688$

4. Result 1: defining $Z_{P, T}$ in type $D$

5. Result 2: $\psi\left(\pi^{-1}\left(Y_{P, T}\right)\right) \subset Z_{P, T}$

6. Result 3: $Z_{P, T} \subset \psi\left(\pi^{-1}\left(Y_{P, T}\right)\right) \quad 701$

7. The Grothendieck ring 711

8. Computing triple intersection numbers 712

9. Computing Pieri coefficients 719

Acknowledgments $\quad 721$

$\begin{array}{ll}\text { References } & 722\end{array}$

\section{Introduction}

When studying the ordinary cohomology of an (isotropic) Grassmannian, a triple intersection number refers to the number of intersection points of three Schubert varieties in general position. By convention, this number is zero when the triple intersection has positive dimension. Algebraically this number is given as the pushforward of the product of three Schubert classes to the cohomology ring of a single point.

MSC2010: primary 14N15; secondary 19E08, 14M15.

Keywords: triple intersection numbers, isotropic Grassmannian, orthogonal Grassmannian, submaximal Grassmannian, Richardson variety, projected Richardson variety, Pieri rule, $K$-theoretic Pieri formula, $K$-theoretic triple intersection. 
Given three Schubert varieties in general position, let $Z$ denote their schemetheoretic triple intersection. The corresponding $K$-theoretic triple intersection number is the sheaf Euler characteristic of $Z$; that is, the pushforward of the product of the three Schubert classes to the Grothendieck ring of a point. We denote this number by $\chi(Z)$. If $Z$ is finite, then, just as in cohomology, $\chi(Z)$ is equal to the number of points in $Z$ (since these finitely many points are reduced, by Kleiman's transversality theorem [1974]). If $Z$ has positive dimension however, then $\chi(Z)$ can be a nonzero (and possibly negative) integer.

In either setting, the triple intersection numbers determine the structure constants for multiplication with respect to the Schubert basis. These structure constants are known as Littlewood-Richardson coefficients, and in ordinary cohomology they are equal to triple intersection numbers. In $K$-theory however, the LittlewoodRichardson coefficients are alternating sums of triple intersection numbers.

An arbitrary Schubert class can be written as an integer polynomial in certain special Schubert classes, which (in cohomology) are closely related to the Chern classes of the tautological quotient bundle on the Grassmannian in question. A triple intersection number is said to be of Pieri-type if one of the three Schubert classes is a special Schubert class. Similarly, a Pieri coefficient refers to a LittlewoodRichardson coefficient occurring in the product of an arbitrary Schubert class and a special Schubert class.

In this paper, we determine $K$-theoretic Pieri-type triple intersection numbers for all isotropic Grassmannians of types $B, C$, and $D$. Our results generalize [Buch and Ravikumar 2012], in which similar calculations are carried out for the cominuscule Grassmannians, that is, for the type- $A$ Grassmannian $\operatorname{Gr}\left(m, \mathbb{C}^{N}\right)$, the maximal odd orthogonal Grassmannian $\mathrm{OG}\left(m, \mathbb{C}^{2 m+1}\right)$, and the Lagrangian Grassmannian $\mathrm{LG}\left(m, \mathbb{C}^{2 m}\right)$.

1A. Methods and results. Let $\omega$ be a skew-symmetric or symmetric nondegenerate bilinear form on $\mathbb{C}^{N}$, where $N \geq 2$. Fix a basis $\boldsymbol{e}_{1}, \ldots, \boldsymbol{e}_{N}$ for $\mathbb{C}^{N}$ that is isotropic in the sense that

$$
\omega\left(\boldsymbol{e}_{i}, \boldsymbol{e}_{j}\right)=\delta_{i+j, N+1} \quad \text { for } 1 \leq i \leq j \leq N .
$$

Note that if $\omega$ is symmetric, then $\omega\left(\boldsymbol{e}_{i}, \boldsymbol{e}_{j}\right)=\delta_{i+j, N+1}$ for all $i$ and $j$ in the integer interval $[1, N]$. If $\omega$ is skew-symmetric (which can only happen when $N$ is even), then $\omega\left(\boldsymbol{e}_{i}, \boldsymbol{e}_{j}\right)=-\delta_{i+j, N+1}$ for $i>j$.

For any subspace $\Sigma \subset \mathbb{C}^{N}$, we define $\Sigma^{\perp}:=\left\{\boldsymbol{w} \in \mathbb{C}^{N}: \omega(\boldsymbol{v}, \boldsymbol{w})=0\right.$ for all $\left.\boldsymbol{v} \in \Sigma\right\}$. We say $\Sigma$ is isotropic if $\Sigma \subset \Sigma^{\perp}$. Given a positive integer $m \leq N / 2$, the isotropic Grassmannian $\operatorname{IG}_{\omega}\left(m, \mathbb{C}^{N}\right)$ is defined as

$$
\mathrm{IG}_{\omega}\left(m, \mathbb{C}^{N}\right):=\left\{\Sigma \in \mathrm{Gr}\left(m, \mathbb{C}^{N}\right): \Sigma \subset \Sigma^{\perp}\right\} .
$$


This projective variety parametrizes isotropic $m$-planes in $\mathbb{C}^{N}$. It is said to have Lie-type $C$ when $\omega$ is skew-symmetric (in which case $N$ is even), Lie-type $B$ when $\omega$ is symmetric and $N$ is odd, and Lie-type $D$ when $\omega$ is symmetric and $N$ is even.

In order to define Schubert varieties in $X:=\operatorname{IG}_{\omega}\left(m, \mathbb{C}^{N}\right)$, we must fix some flags on $\mathbb{C}^{N}$. We define the standard flag $E_{\bullet}$ on $\mathbb{C}^{N}$ by $E_{j}:=\left\langle\boldsymbol{e}_{1}, \ldots, \boldsymbol{e}_{j}\right\rangle$, the span of the first $j$ basis vectors. In types $B$ and $C$, we define the opposite flag $E_{\bullet}^{\text {op }}$ by $E_{j}^{\text {op }}:=\left\langle\boldsymbol{e}_{N+1-j}, \ldots, \boldsymbol{e}_{N}\right\rangle$, the span of the last $j$ basis vectors. A more complicated type- $D$ definition is given in Section 3 .

Given $\Sigma \in X$, the Schubert symbol of $\Sigma$ relative to $E_{\bullet}$,

$$
\mathfrak{s}(\Sigma):=\left\{c \in[1, N]: \Sigma \cap E_{c} \supsetneq \Sigma \cap E_{c-1}\right\},
$$

records the steps $c$ in $E_{\text {. }}$ at which the intersection $\Sigma \cap E_{c}$ jumps dimension. Note that the set $\mathfrak{s}(\Sigma)$ has cardinality $m$, and that if $c \in \mathfrak{s}(\Sigma)$ then $N+1-c \notin \mathfrak{s}(\Sigma)$, since $\Sigma$ is isotropic. In general, a subset $P \subset[1, N]$ of cardinality $m$ is a Schubert symbol $^{1}$ if $c+d \neq N+1$ for any $c, d \in P$. We let $\Omega(X)$ denote the set of all Schubert symbols for $X$.

Given a Schubert symbol $P$, we define the Schubert variety $X_{P}:=X_{P}\left(E_{\bullet}\right)$ to be the closure in $X$ of the Schubert cell $X_{P}^{\circ}\left(E_{\bullet}\right):=\{\Sigma \in X: \mathfrak{s}(\Sigma)=P\}$. We say $X_{P}$ is a Schubert variety relative to the flag $E_{\text {. }}$. We also define the opposite Schubert variety $X^{P}$ to be the unique Schubert variety relative to the opposite flag $E_{\bullet}^{\text {op }}$ that intersects $X_{P}$ at a single point. For the special Schubert varieties, we adopt an additional indexing convention, writing $X_{(r)}$ to denote the special Schubert variety of codimension $r$ in $X$. Given Schubert symbols $P$ and $T$, we write $T \preceq P$ if $X_{T} \subset X_{P}$. The resulting partial order on the set of Schubert symbols, known as the Bruhat order, is described combinatorially in Sections 2 and 3.

The transverse intersection of two Schubert varieties is known as a Richardson variety. Associated to Schubert symbols $P$ and $T$ we have a Richardson variety $Y_{P, T}:=X_{P} \cap X^{T}$, which is nonempty if and only if $T \preceq P$. Since $\left[\mathrm{O}_{X_{P}}\right] \cdot\left[\mathrm{O}_{X^{T}}\right]=$ $\left[{ }^{O} Y_{P, T}\right]$ (see, e.g., [Brion 2005]), the $K$-theoretic Pieri-type triple intersection numbers can be written

$$
\chi\left(\left[\mathrm{O}_{Y_{P, T}}\right] \cdot\left[\mathrm{O}_{X_{(r)}}\right]\right),
$$

where $\chi: K(X) \rightarrow \mathbb{Z}$ is the sheaf Euler characteristic map. These numbers are nonzero only when $T \preceq P$.

We can reinterpret this triple intersection number by means of the following incidence relation, which consists of the two-step isotropic flag variety $\operatorname{IF}_{\omega}\left(1, m, \mathbb{C}^{N}\right)$ whose natural projections we denote by $\psi$ (to the Grassmannian $I G_{\omega}\left(1, \mathbb{C}^{N}\right)$ ) and

${ }^{1}$ Schubert symbols are sometimes known as jump sequences, and in [Buch et al. 2009] they are referred to as index sets. 
$\pi$ (to the Grassmannian $I G_{\omega}\left(m, \mathbb{C}^{N}\right)$ ).

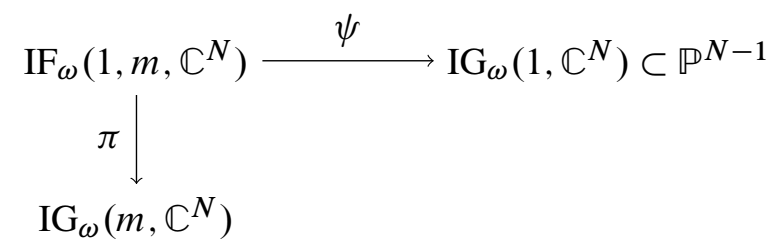

In particular, we make use of the projected Richardson variety $\psi\left(\pi^{-1}\left(Y_{P, T}\right)\right) \subset$ $\mathbb{P}^{N-1}$, which is the (projectivization of the) union of all $m$-planes in the Richardson variety $Y_{P, T}$. Projected Richardson varieties like $\psi\left(\pi^{-1}\left(Y_{P, T}\right)\right)$ have a number of nice geometric properties. He and Lam [2011] relate these varieties to the $K$-theory of affine Grassmannians, and it has been proved by Billey and Coskun [2012], and by Knutson, Lam, and Speyer [Knutson et al. 2014], that they are Cohen-Macaulay with rational singularities and that the projection map is cohomologically trivial, in the sense that $\psi_{*}\left[0_{\pi^{-1}\left(Y_{P, T}\right)}\right]=\left[\mathrm{O}_{\psi\left(\pi^{-1}\left(Y_{P, T}\right)\right)}\right]$. By this last fact, along with the projection formula, the calculation of the triple intersection number (2) amounts to showing that $\psi\left(\pi^{-1}\left(Y_{P, T}\right)\right)$ is a complete intersection in $\mathbb{P}^{N-1}$ and determining the equations that define it.

A description of the projected Richardson variety $\psi\left(\pi^{-1}\left(Y_{P, T}\right)\right)$ is carried out in [Buch et al. 2009], but in the special case that the Schubert symbols $P$ and $T$ satisfy a relation $P \rightarrow T$. Roughly speaking, this relation signifies that $T$ shows up in some cohomological Pieri product involving $P$. The relation $P \rightarrow T$ requires that $T \preceq P$, and $T \preceq P$ is a more general condition. We note that for $P \not \neg T$, the $K$-theoretic triple intersection numbers $\chi\left(\left[{ }_{Y_{P, T}}\right] \cdot\left[{ } X_{(r)}\right]\right)$ need not vanish (in contrast to the cohomological triple intersection numbers), and are therefore essential ingredients for the $K$-theoretic Pieri coefficients.

When $X$ is a Grassmannian of Lie type $B$ or $C$, Buch, Kresch, and Tamvakis [Buch et al. 2009] define a complete intersection $Z_{P, T} \subset \mathbb{P}^{N-1}$ for Schubert symbols $T \preceq P$, and prove that the projected Richardson variety $\psi\left(\pi^{-1}\left(Y_{P, T}\right)\right)$ is contained in it. They attempt to extend the definition of $Z_{P, T}$ to the type- $D$ Grassmannian, but use an erroneous definition of Schubert varieties, resulting in a definition of $Z_{P, T}$ that only makes sense in the special case that $P \rightarrow T$.

The first result of this paper, presented in Section 4, is to provide a corrected definition of $Z_{P, T}$ in the general setting that $T \preceq P$, and to show that it is a complete intersection of linear and quadratic hypersurfaces. This process involves new combinatorics of Schubert symbols, such as the notion of an exceptional cut.

The second result, presented in Section 5, is that $\psi\left(\pi^{-1}\left(Y_{P, T}\right)\right) \subset Z_{P, T}$ for any Schubert symbols $T \preceq P$ in a Grassmannian of Lie-type $B, C$, or $D$. This result generalizes [Buch et al. 2009, Lemma 5.1], in which this statement is proved in types $B$ and $C$ only. 
The third result, presented in Section 6, is that given a type- $B, C$, or $D$ Grassmannian and arbitrary Schubert symbols $T \preceq P$, we have $Z_{P, T} \subset \psi\left(\pi^{-1}\left(Y_{P, T}\right)\right)$. We prove this result by constructing a smaller Richardson variety contained in $Y_{P, T}$ that projects surjectively onto $Z_{P, T}$.

Combining these results, we arrive at the main theorem of this paper:

Theorem 1.1. Let $X$ be a Grassmannian of Lie-type $B, C$, or D. For any Schubert symbols $T \preceq P$, we have $Z_{P, T}=\psi\left(\pi^{-1}\left(Y_{P, T}\right)\right)$.

By Theorem 1.1, we know exactly which equations define the projected Richardson variety in all three Lie types. These equations allow for a pleasant calculation of the triple intersection numbers, which we carry out in Section 8. In Section 9, we describe how $K$-theoretic Pieri coefficients are calculated as alternating sums of these triple intersection numbers. Taken together, the results of this paper complete the story of Pieri-type triple intersection numbers for Grassmannians. We hope this approach will soon lead to a positive Pieri formula.

\section{Preliminaries 1: types $B$ and $C$}

2A. Schubert symbols. Let $X:=\mathrm{IG}_{\omega}\left(m, \mathbb{C}^{N}\right)$ be a Grassmannian of type $C$ or $B$, where $N:=2 n$ or $N:=2 n+1$, depending on whether $X$ is of type $C$ or $B$ respectively. In the former case, we will also denote $X$ by $\operatorname{SG}(m, 2 n)$ and refer to it as a symplectic Grassmannian. In the latter case, we will also denote $X$ by $\mathrm{OG}(m, 2 n+1)$ and refer to it as an odd orthogonal Grassmannian. Recall that for Schubert symbols $T$ and $P$ in $\Omega(X)$, the relation $T \preceq P$ signifies that $X_{T} \subset X_{P}$. This partial order on the set of Schubert symbols has a simple combinatorial description.

Given Schubert symbols $T=\left\{t_{1}<\cdots<t_{m}\right\}$ and $P=\left\{p_{1}<\cdots<p_{m}\right\}$, we write $T \leq P$ whenever $t_{i} \leq p_{i}$ for $1 \leq i \leq m$. By [Buch et al. 2009, Proposition 4.1] we have the following lemma:

Lemma 2.1. Provided $X$ is of Lie-type $B$ or $C$, we have $T \leq P$ if and only if $T \preceq P$.

For any Schubert symbol $P \in \Omega(X)$, let $\bar{P}=\{c \in[1, N]: N+1-c \in P\}$ and $[P]=P \cup \bar{P}$. Also let $|P|$ denote the codimension of the Schubert variety $X_{P}$ in $X$.

For each Schubert symbol $P$, there is a unique dual symbol $P^{\vee}$ with the property that for any Schubert symbol $T, X_{P}\left(E_{\bullet}\right) \cap X_{T}\left(E_{\bullet}^{\mathrm{op}}\right)$ is equal to a single point if and only if $T=P^{\vee}$. The opposite Schubert symbol $X^{P}$ defined in the introduction is therefore equal to $X_{P^{\vee}}\left(E_{\bullet}^{\mathrm{op}}\right)$. The following lemma, from [Buch et al. 2009, Proposition 4.2], gives a simple description the dual symbol $P^{\vee}$ :

Lemma 2.2. Provided $X$ is of Lie-type $B$ or $C$, we have $P^{\vee}=\bar{P}$ for all Schubert symbols $P \in \Omega(X)$. 
2B. Richardson diagrams. It is a well-known fact (following from Borel's fixedpoint theorem [1956]) that $T \preceq P$ if and only if $X_{P} \cap X^{T}$ is nonempty. This variety $Y_{P, T}:=X_{P} \cap X^{T}$ is connected, and hence reduced and irreducible by Kleiman's transversality theorem [1974] (see also [Richardson 1992]). It is known as a Richardson variety.

Given Schubert symbols $T \leq P$, we define the Richardson diagram $D(P, T)=$ $\left\{(j, c): t_{j} \leq c \leq p_{j}\right\}$, which we represent as an $m \times N$ matrix with a $*$ for every entry in $D(P, T)$ and zeros elsewhere. We say a matrix $\left(a_{i, j}\right)$ has shape $D(P, T)$ if its dimensions are $m \times N$ and $a_{j, c}=0$ for all $(j, c) \notin D(P, T)$. Given a matrix of shape $D(P, T)$ whose row vectors are independent and orthogonal, its rowspace will be an element of $Y_{P, T}$.

Example 2.3. Any rank- $m$ matrix of shape $D(P, P)$ will have rowspace $\Sigma_{P}:=$ $\left\langle\boldsymbol{e}_{p_{1}}, \ldots, \boldsymbol{e}_{p_{m}}\right\rangle$, which is the only element of $Y_{P, P}$.

Example 2.4. Suppose $P=\{2,3,4,10\}$ and $T=\{1,2,4,6\}$ in $\operatorname{SG}(4,10)$. Suppose $\left(a_{i, j}\right)$ is a rank- $m$ matrix of shape $D(P, T)$. The rowspace of $\left(a_{i, j}\right)$ will be in $Y_{P, T}$ if and only if $a_{1,1} a_{4,10}+a_{1,2} a_{4,9}=0, a_{2,2} a_{4,9}+a_{2,3} a_{4,8}=0$, and $a_{4,7}=0$. We leave it to the reader to write down explicit entries satisfying these equations. The diagram $D(P, T)$ is

$$
\left(\begin{array}{llllllllll}
* & * & 0 & 0 & 0 & 0 & 0 & 0 & 0 & 0 \\
0 & * & * & 0 & 0 & 0 & 0 & 0 & 0 & 0 \\
0 & 0 & 0 & * & 0 & 0 & 0 & 0 & 0 & 0 \\
0 & 0 & 0 & 0 & 0 & * & * & * & * & *
\end{array}\right) .
$$

Given a Schubert symbol $P=\left\{p_{1}, \ldots, p_{m}\right\}$, let $p_{0}=0$ and $p_{m+1}=N+1$. We won't consider these as actual elements in the Schubert symbol $P$, but the notation will be useful. Define a visible cut through $D(P, T)$ to be any integer $c \in[0, N]$ such that no row of $D(P, T)$ contains stars in both column $c$ and column $c+1$; i.e., such that $p_{i} \leq c<t_{i+1}$ for some $i$. We will consider $c=0$ and $c=N$ to be visible cuts. Define an apparent $c u t$ to be any integer $c \in[0, N]$ such that $c$ or $N-c$ is a visible cut. In types $B$ and $C$ we define a $c u t$ in $D(P, T)$ to be synonymous with an apparent cut. Let $\mathscr{C}_{P, T}$ be the set of cuts in $D(P, T)$.

An integer $c$ is a zero column of $D(P, T)$ if $p_{j}<c<t_{j+1}$ for some $j$, since in this case column $c$ of $D(P, T)$ has no stars. An entry $(j, c)$ in $D(P, T)$ is a lone star if either

(i) $c \in T$ and $c$ is a cut in $D(P, T)$, or

(ii) $c \in P$ and $c-1$ is a cut in $D(P, T)$.

The simplest example of a lone star occurs when $t_{j}=p_{j}=c$ for some $j$. In this case row $j$ and column $c$ of $D(P, T)$ each contain a single star at $(j, c)$. We define the set $\mathscr{L}_{P, T} \subset[1, N]$ to be the set of integers $c$ such that either 
(i) $c$ is a zero column in $D(P, T)$, or

(ii) there exists a lone star in column $N+1-c$.

Finally, we define the set

$$
\mathscr{2}_{P, T}:=\left\{\begin{array}{cl}
{[0, n] \cap \mathscr{C}_{P, T}} & \text { if } X \text { is of type } C, \\
\left([0, n] \cap \mathscr{C}_{P, T}\right) \cup\{n+1\} & \text { if } X \text { is of type } B .
\end{array}\right.
$$

Example 2.5. Continuing with Example 2.4, the set of cuts $\mathscr{C}_{P, T}$ is equal to $\{0,3,4,5,6,7,10\}$. Of these, $0,3,4,5$, and 10 are visible cuts. Furthermore, 5 is a zero column, $(3,4)$ is a lone star, $\mathscr{L}_{P, T}=\{5,7\}$, and $\mathscr{Q}_{P, T}=\{0,3,4,5\}$.

In types $B$ and $C$, lone stars take a particularly simple form. Namely:

Proposition 2.6. Let $X$ be an isotropic Grassmannian of Lie-type B or C. Suppose $(j, c)$ is a lone star in $D(P, T)$. If $c=t_{j}$ and $t_{j}$ is an apparent cut, or if $c=p_{j}$ and $p_{j}-1$ is an apparent cut, then either $N+1-c$ is a zero column or $t_{j}=p_{j}=c$.

Proof. Suppose $c=t_{j}$ is an apparent cut in $D(P, T)$, and that $N+1-c$ is not a zero column. Since $N+1-c$ is not in $T$ and not a zero-column, it follows that $N-c$ is not a visible cut. But then $c$ must be a visible cut, so $c=p_{j}$. A similar argument holds if we start by assuming $c=p_{j}$.

Since zero columns are flanked by cuts, we have the following immediate result:

Corollary 2.7. In types $B$ and $C$, if $c \in \mathscr{L}_{P, T}$ then $c$ and $c-1$ are both cuts.

2C. The projected Richardson variety. We now define a subvariety of $\mathbb{P}^{N-1}$ that will play a key role in the calculation of triple intersection numbers. Let $x_{1}, \ldots, x_{N} \in\left(\mathbb{C}^{N}\right)^{*}$ be the dual basis to the isotropic basis $\boldsymbol{e}_{1}, \ldots, \boldsymbol{e}_{N} \in \mathbb{C}^{N}$. Let $f_{0}=0$, and for $1 \leq c \leq n$ let $f_{c}=x_{1} x_{N}+\cdots+x_{c} x_{N+1-c}$. For example, $f_{1}=x_{1} x_{N}$ and $f_{2}=x_{1} x_{N}+x_{2} x_{N-1}$. In addition, if $X$ is type $B$, let $f_{n+1}=x_{1} x_{2 n+1}+\cdots+x_{n} x_{n+2}+\frac{1}{2} x_{n+1}^{2}$. Given Schubert symbols $T \preceq P$, let $Z_{P, T} \subset \mathbb{P}^{N-1}$ denote the subvariety defined by the vanishing of the polynomials $\left\{f_{c} \mid c \in \mathscr{Q}_{P, T}\right\} \cup\left\{x_{c} \mid c \in \mathscr{L}_{P, T}\right\}$. We note that, in the type- $B$ case, $Z_{P, T}$ must satisfy the equation $f_{n+1}=0$ and hence lie in $\mathrm{OG}(1,2 n+1)$, the quadric hypersurface of isotropic lines in $\mathbb{P}^{2 n}$.

In fact, $Z_{P, T}$ is a complete intersection in $\mathbb{P}^{N-1}$ cut out by the polynomials:

(a) $f_{d}-f_{c}=x_{c+1} x_{N-c}+\cdots+x_{d} x_{N+1-d}$ if $c$ and $d$ are consecutive elements of $2_{P, T}$ such that $d-c \geq 2$.

(b) $x_{c}$ if $c \in \mathscr{L}_{P, T}$.

We will prove this fact for all three Lie types in Proposition 4.15.

Recall that we have projections $\pi$ and $\psi$ from the flag variety $\operatorname{IF}_{\omega}\left(1, m, \mathbb{C}^{N}\right)$ to $X$ and $\operatorname{IG}_{\omega}\left(1, \mathbb{C}^{N}\right)$, respectively. The variety $\pi^{-1}\left(Y_{P, T}\right)$ is a Richardson variety in $\operatorname{IG}_{\omega}\left(1, m, \mathbb{C}^{N}\right)$, and its image $\psi\left(\pi^{-1}\left(Y_{P, T}\right)\right)$ is known as a projected Richardson 
variety. We shall prove that the projected Richardson variety $\psi\left(\pi^{-1}\left(Y_{P, T}\right)\right)$ is in fact equal to $Z_{P, T}$. One inclusion is straightforward:

Lemma 2.8. Given Schubert symbols $T \preceq P$ for a Grassmannian $X$ of Lie-type $B$ or $C$, we have $\psi\left(\pi^{-1}\left(Y_{P, T}\right)\right) \subset Z_{P, T}$.

A proof of Lemma 2.8 can be found in [Buch et al. 2009, Lemma 5.1]. This proof is correct for types $B$ and $C$, but does not go through in type $D$ due to an erroneous definition of the Bruhat order. We supply a corrected proof for all three Lie types in Section 5.

Example 2.9. Continuing with Example 2.5, suppose $\boldsymbol{M}$ is a matrix with shape $D(P, T)$ and independent, isotropic row vectors. Note that any vector in the rowspace of $\boldsymbol{M}$ must satisfy the quadratic equation $x_{1} x_{10}+x_{2} x_{9}+x_{3} x_{8}=0$ and the linear equations $x_{5}=0$ and $x_{7}=0$, which are precisely the equations defining $Z_{P, T}$. By Lemma 2.8, any vector contained in an $m$-plane $\Sigma \in Y_{P, T}$ satisfies these equations.

\section{Preliminaries 2: type $D$}

Consider $\mathbb{C}^{2 n+2}$, endowed with a nondegenerate symmetric bilinear form. Let $X:=\mathrm{OG}(m, 2 n+2)$ denote the even orthogonal Grassmannian of isotropic $m$ planes in $\mathbb{C}^{2 n+2}$, where $1 \leq m \leq n+1$. In this section we describe the Bruhat order for even orthogonal Grassmannians, which is more complicated than in types $B$ and $C$. We note that definition (1) implies that $\mathrm{OG}(n+1,2 n+2)$ is disconnected. Although we won't go into it here, this fact can help to give a geometric intuition behind the Bruhat order on even orthogonal Grassmannians (see [Ravikumar 2013, Chapter 5] for a detailed description).

For any Schubert symbol $P \in \Omega(X)$, let $\bar{P}=\{c \in[1,2 n+2]: 2 n+3-c \in P\}$ and $[P]=P \cup \bar{P}$. As before, let $|P|$ denote the codimension of the Schubert variety $X_{P}$ in $X$. We define $\mathfrak{t}(P) \in\{0,1,2\}$ as follows. If $n+1 \in[P]$, then we let $\mathfrak{t}(P)$ be congruent mod 2 to the number of elements in $[1, n+1] \backslash P$. In other words, if $\#([1, n+1] \backslash P)$ is even then $\mathfrak{t}(P)=0$, and if $\#([1, n+1] \backslash P)$ is odd then $\mathfrak{t}(P)=1$. Finally, if $\{n+1, n+2\} \cap P=\varnothing$, we set $\mathfrak{t}(P)=2$. $^{2}$

Proposition 3.1 [Buch et al. 2013, Proposition A.2]. Given Schubert symbols $P$ and $T$ in $\Omega(\mathrm{OG}(m, 2 n+2))$, we have $T \preceq P$ if and only if

(i) $T \leq P$, and

(ii) if there exists $c \in[1, n]$ such that $[c+1, n+1] \subset[P] \cap[T]$ and $\# P \cap[1, c]=$ $\# T \cap[1, c]$, then we have $\mathfrak{t}(P)=\mathfrak{t}(T)$.

${ }^{2} \mathfrak{t}(P)$ differs slightly from the function type $(P)$ in [Buch et al. 2013]. Namely, $\operatorname{type}(P) \equiv$ $\mathfrak{t}(P)+1(\bmod 3)$. 
By Proposition 3.1, the type- $D$ Bruhat order is not simply the $\leq$ ordering. The following example illustrates the difference.

Example 3.2. The $\preceq$ partial order is shown below for the Schubert symbols on $\operatorname{OG}\left(2, \mathbb{C}^{6}\right)$, which are colored by type. Notice that there are six "missing" edges, which would have occurred had we used the (incorrect) $\leq$ ordering.

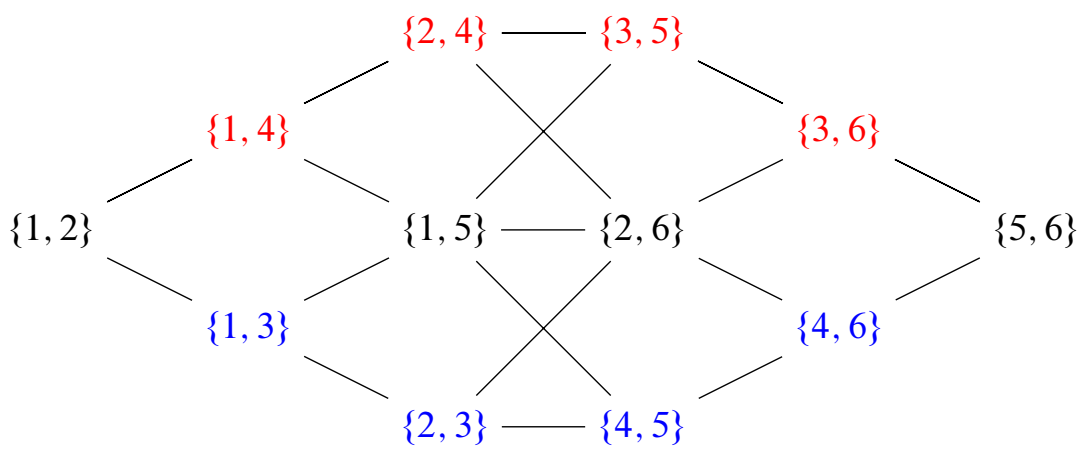

We define the opposite flag $E_{\bullet}^{\mathrm{op}}$ by $E_{j}^{\mathrm{op}}=\left\langle\boldsymbol{e}_{2 n+3-j}, \ldots, \boldsymbol{e}_{2 n+2}\right\rangle$ for $j \neq n+1$ and

$$
E_{n+1}^{\mathrm{op}}= \begin{cases}\left\langle\boldsymbol{e}_{n+2}, \boldsymbol{e}_{n+3}, \ldots, \boldsymbol{e}_{2 n+2}\right\rangle & \text { if } n \text { is odd } \\ \left\langle\boldsymbol{e}_{n+1}, \boldsymbol{e}_{n+3}, \ldots, \boldsymbol{e}_{2 n+2}\right\rangle & \text { if } n \text { is even }\end{cases}
$$

This definition guarantees that $E_{\bullet}$ and $E_{\bullet}^{\text {op }}$ lie in the same connected component of the variety of complete isotropic flags on $\mathbb{C}^{2 n+2}$ (endowed with a nondegenerate symmetric bilinear form), which is disconnected.

Let $\iota$ be the permutation of $\{1, \ldots, 2 n+2\}$ that interchanges $n+1$ and $n+2$ and leaves all other numbers fixed. Given a type- $D$ Schubert symbol $P=\left\{p_{1}, \ldots, p_{m}\right\}$, let $\iota(P)=\left\{\iota\left(p_{1}\right), \ldots, \iota\left(p_{m}\right)\right\}$. From [Buch et al. 2009, p. 43], we have the following description of the dual symbol $P^{\vee}$ :

Lemma 3.3. Given a Schubert symbol $P \in \Omega(\mathrm{OG}(m, 2 n+2))$, we have

$$
P^{\vee}= \begin{cases}\bar{P} & \text { when } n \text { is odd } \\ \iota(\bar{P}) & \text { when } n \text { is even } .\end{cases}
$$

If the type- $D$ definitions of opposite flags and dual Schubert symbols appear confusing, the following observation may offer some relief:

Observation 3.4. Let $X:=\mathrm{IG}_{\omega}(m, N)$ be a Grassmannian of type $B, C$, or $D$. For any Schubert symbol $P$, we have

$$
X_{P^{\vee}}^{\circ}\left(E_{\bullet}^{\mathrm{op}}\right)=\left\{\Sigma \in \mathrm{IG}: \Sigma \cap\left\langle\boldsymbol{e}_{p_{i}}, \ldots, \boldsymbol{e}_{N}\right\rangle \supsetneq \Sigma \cap\left\langle\boldsymbol{e}_{p_{i}+1}, \ldots, \boldsymbol{e}_{N}\right\rangle\right\} .
$$


Observation 3.4 is obvious unless we are working in $\mathrm{OG}(m, 2 n+2)$ and $n$ is even. We illustrate that case in the following example:

Example 3.5. Consider $\mathrm{OG}(1,6)$, and let $P=\{4\}$. Then $P^{\vee}=\{4\}, E_{3}^{\mathrm{op}}=$ $\left\langle\boldsymbol{e}_{3}, \boldsymbol{e}_{5}, \boldsymbol{e}_{6}\right\rangle$, and $E_{4}^{\mathrm{op}}=\left\langle\boldsymbol{e}_{3}, \boldsymbol{e}_{4}, \boldsymbol{e}_{5}, \boldsymbol{e}_{6}\right\rangle$. By definition,

$$
X_{P^{\vee}}^{\circ}\left(E_{\bullet}^{\mathrm{op}}\right)=\left\{\Sigma \in \mathrm{IG}: \Sigma \cap E_{4}^{\mathrm{op}} \supsetneq \Sigma \cap E_{3}^{\mathrm{op}}\right\},
$$

which is equal to the set of points in $\mathbb{P}^{5}$ of the form $\langle(0,0,0,1, *, *)\rangle$, in agreement with Observation 3.4.

By Observation 3.4, any element of the Schubert cell $X_{P^{\vee}}^{\circ}\left(E_{\bullet}^{\text {op }}\right)$ is the rowspace of an isotropic $m \times N$ matrix $\left(a_{i, j}\right)$ with $a_{i, p_{i}}=1$ for $1 \leq i \leq m$ and $a_{i, j}=0$ for $j<p_{i}$.

Example 3.6. Consider $\operatorname{OG}(3,10)$, and let $P=\{1,4,5\}$. In this case, $P^{\vee}=$ $\{5,7,10\}$. We can write any element of $X_{P^{\vee}}^{\circ}\left(E_{\bullet}^{\text {op }}\right)$ as the rowspace of a matrix of the form

$$
\left(\begin{array}{llllllllll}
1 & * & * & * & * & * & * & * & * & * \\
0 & 0 & 0 & 1 & * & * & * & * & * & * \\
0 & 0 & 0 & 0 & 1 & 0 & * & * & * & *
\end{array}\right) .
$$

Thus $X_{T \vee}\left(E_{\bullet}^{\mathrm{op}}\right)=X^{T}$ for any Schubert symbol $T$, since $X_{T} \cap X_{T^{\vee}}\left(E_{\bullet}^{\mathrm{op}}\right)$ is a single point. We define the Richardson variety $Y_{P, T}:=X_{P} \cap X^{T}$. As before, $Y_{P, T} \neq \varnothing$ if and only if $T \preceq P$. We define the Richardson diagram $D(P, T):=$ $\left\{(j, c): t_{j} \leq c \leq p_{j}\right\}$ for any Schubert symbols $T \leq P$. This definition holds when $T \npreceq P$, but in this case there cannot exist a matrix of shape $D(P, T)$ whose row vectors are independent and orthogonal (a fact we shall prove in Proposition 4.12).

Example 3.7. There are no matrices $\left(a_{i, j}\right)$ of shape $D(\{2,5,7,8\},\{1,3,4,6\})$ whose rows span an element of $\operatorname{OG}(4,10)$, because the isotropic relations on the entries are inconsistent:

$$
\left(\begin{array}{cccccccccc}
a_{1,1} & a_{1,2} & 0 & 0 & 0 & 0 & 0 & 0 & 0 & 0 \\
0 & 0 & a_{2,3} & a_{2,4} & a_{2,5} & 0 & 0 & 0 & 0 & 0 \\
0 & 0 & 0 & a_{3,4} & a_{3,5} & a_{3,6} & a_{3,7} & 0 & 0 & 0 \\
0 & 0 & 0 & 0 & 0 & a_{4,6} & a_{4,7} & a_{4,8} & 0 & 0
\end{array}\right) .
$$

We leave it to the reader to verify this fact, as well as the fact that $\{1,3,4,6\} \not ૂ$ $\{2,5,7,8\}$ in type $D$.

\section{Result 1: defining $Z_{P, T}$ in type $D$}

Let $X:=\mathrm{OG}(m, 2 n+2)$ be a type- $D$ Grassmannian, let $N:=2 n+2$, and let $T \preceq P$ be Schubert symbols in $\Omega(X)$. Visible cuts, apparent cuts, lone stars, and zero columns in $D(P, T)$ are defined exactly as in types $B$ and $C$. Similarly, $\mathscr{C}_{P, T}$ 
continues to denote the set of all cuts in $D(P, T)$, and $\mathscr{L}_{P, T}$ continues to denote the set of integers $c \in[1,2 n+2]$ such that either $c$ is a zero column or column $2 n+3-c$ contains a lone star. However, in order to define the subvariety $Z_{P, T} \subset \mathbb{P}^{2 n+1}$, we must define a new type of cut in $D(P, T)$.

4A. Exceptional cuts. If for some $i$ we have $p_{i}=n+2 \leq t_{i+1}$ or $t_{i}=n+1 \geq p_{i-1}$, we let $n+1$ be a cut in $D(P, T)$, which we will refer to as an exceptional center cut. This cut will induce a lone star in column $n+2$ or $n+1$ respectively.

Example 4.1. $P=\{2,4\}$ and $T=\{1,2\}$ in $\mathrm{OG}(2,6) . D(P, T)$ is shown below, and has an exceptional center cut. As a result, $(2,4)$ is a lone star, and $3 \in \mathscr{L}_{P, T}$.

$$
\left(\begin{array}{lll|lll}
* & * & 0 & 0 & 0 & 0 \\
0 & * & * & * & 0 & 0
\end{array}\right)
$$

There are additional exceptional cuts in $D(P, T)$. Let $c \in[1, n]$ be a cut candidate if $[c+1, n+1] \subset[P] \cap[T]$ and $\#(T \cap[1, c])=\#(P \cap[1, c])+1$. If $\mathfrak{t}(T) \neq \mathfrak{t}(P)$, then $c$ and $N+1-c$ will also be cuts in $D(P, T)$ for each cut candidate $c$. We'll refer to these as exceptional cuts as well. We give several examples of diagrams with exceptional cuts, as the definition is somewhat complicated.

Example 4.2. $P=\{3,6\}$ and $T=\{2,3\}$ in $\mathrm{OG}(2,6) . D(P, T)$ is shown below, and $\mathscr{C}=\{0,1,2,3,4,5,6\}$. Of these, 2,3 (the center cut), and 4 are exceptional cuts. $(1,2),(1,3)$, and $(2,3)$ are all lone stars, and $\mathscr{L}_{P, T}=\{1,4,5\}$.

$$
\left(\begin{array}{ll|l|l|ll}
0 & * & * & 0 & 0 & 0 \\
0 & 0 & * & * & * & *
\end{array}\right)
$$

Example 4.3. $P=\{3,4,7\}$ and $T=\{1,3,4\}$ in $\mathrm{OG}(3,8) . D(P, T)$ is shown below, and $\mathscr{C}=\{0,1,2,3,4,5,6,7,8\}$. Of these, 2, 3, 4 (the center cut), 5, and 6 are exceptional cuts. By finding all the lone stars, one can check that $\mathscr{L}_{P, T}=\{2,5,6,8\}$.

$$
\left(\begin{array}{cc|c|c|c|c|cc}
* & * & * & 0 & 0 & 0 & 0 & 0 \\
0 & 0 & * & * & 0 & 0 & 0 & 0 \\
0 & 0 & 0 & * & * & * & * & 0
\end{array}\right) .
$$

Example 4.4. $P=\{4,6,8\}$ and $T=\{1,3,5\}$ in $\operatorname{OG}(3,8) . D(P, T)$ is shown below, and $\mathscr{C}=\{0,2,6,8\}$. Of these, 2 and 6 are exceptional cuts, and $\mathscr{L}_{P, T}=\varnothing$.

$$
\left(\begin{array}{cc|cccc|cc}
* & * & * & * & 0 & 0 & 0 & 0 \\
0 & 0 & * & * & * & * & 0 & 0 \\
0 & 0 & 0 & 0 & * & * & * & *
\end{array}\right) .
$$


Example 4.5. $P=\{4,5,8,9\}$ and $T=\{1,3,4,6\}$ in $\operatorname{OG}(4,10) . D(P, T)$ is shown below, and $\mathscr{C}=\{0,1,2,8,9,10\}$. Of these, 2 and 8 are exceptional cuts, and $\mathscr{L}_{P, T}=\{2,10\}$.

$$
\left(\begin{array}{cc|cccccc|cc}
* & * & * & * & 0 & 0 & 0 & 0 & 0 & 0 \\
0 & 0 & * & * & * & 0 & 0 & 0 & 0 & 0 \\
0 & 0 & 0 & * & * & * & * & * & 0 & 0 \\
0 & 0 & 0 & 0 & 0 & * & * & * & * & 0
\end{array}\right) .
$$

We relate certain features of the Richardson diagram $D(P, T)$ to the type- $D$ Bruhat order and to the existence of exceptional cuts.

Lemma 4.6. For any Schubert symbol $P$, the following conditions are equivalent:

(1) $[c+1, n+1] \subset[P]$.

(2) $\#([c+1, N-c] \cap P)=n+1-c$.

Proof. Note that $n+1-c=\#([c+1, n+1])$. Because $P$ is an isotropic Schubert symbol, there can be at most $n+1-c$ elements in $[c+1, N-c] \cap P$. Since $[c+1, n+1] \subset[P]$, there are at least that many.

Lemma 4.7. Given Schubert symbols $T \leq P$ such that $[c+1, n+1] \subset[T] \cap[P]$, we have

$$
\#([1, c] \cap T)-\#([1, c] \cap P)=\#([N+1-c, N] \cap P)-\#([N+1-c, N] \cap T) .
$$

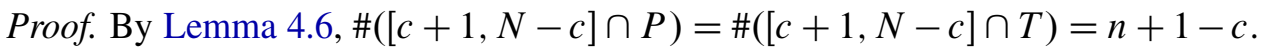
It follows that

$$
\begin{aligned}
\#([1, c] \cap P)+\#([N+1-c, N] & \cap P) \\
& =m-(n+1-c) \\
& =\#([1, c] \cap T)+\#([N+1-c, N] \cap T) .
\end{aligned}
$$

Lemma 4.7 says that whenever $[c+1, n+1] \subset[T] \cap[P]$, the number of rows crossing from column $c$ to column $c+1$ of $D(P, T)$ is equal to the number of rows crossing from column $N-c$ to column $N+1-c$ of $D(P, T)$. We therefore have the following corollary:

Corollary 4.8. Given $c \in[1, n]$, suppose $[c+1, n+1] \subset[T] \cap[P]$ and $\mathfrak{t}(P) \neq \mathfrak{t}(T)$ for Schubert symbols $T \leq P$.

(1) The first four of the following statements are equivalent, and any of them implies the last:

- $\#([1, c] \cap T)=\#([1, c] \cap P)$.

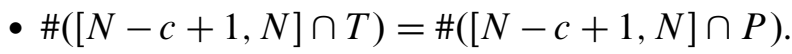

- $c$ is a visible cut in $D(P, T)$. 
- $N-c$ is a visible cut in $D(P, T)$.

- $T \not L P$.

(2) The following statements are equivalent:

- $c$ and $N-c$ are exceptional cuts in $D(P, T)$.

- $\#([1, c] \cap T)=\#([1, c] \cap P)+1$.

- $\#([N-c+1, N] \cap P)=\#([N-c+1, N] \cap T)+1$.

- $D(P, T)$ has exactly one row crossing from column c to column $c+1$.

- $D(P, T)$ has exactly one row crossing from column $N-c$ to column $N-c+1$.

We finish this section by proving that several important properties of Richardson diagrams carry over to the type- $D$ case. In particular, we extend Corollary 2.7 to type $D$, and then prove in Corollary 4.13 that $(P \cup T) \cap \mathscr{L}_{P, T}=\varnothing$ (a fact that is obvious in types $B$ and $C$ ). Once these facts are established, we will be ready to define $Z_{P, T}$.

First we observe that, for any $T \leq P, D(P, T)$ and $D(\bar{T}, \bar{P})$ have the same cut candidates, by Lemma 4.7. It follows that:

Observation 4.9. $180^{\circ}$ rotation of the diagram $D(P, T)$ preserves all cuts, including exceptional cuts. In other words, $\mathscr{C}_{P, T}=\mathscr{C}_{\bar{T}, \bar{P}}$.

We can now prove the type- $D$ version of Corollary 2.7:

Proposition 4.10. In type $D$, if $c \in \mathscr{L}_{P, T}$, then $c$ and $c-1$ are both in $\mathscr{C}_{P, T}$.

Proof. If $c$ is a zero column then the result is clear. Otherwise, it must be the case that $(i, N+1-c)$ is a lone star for some $i$. By Observation 4.9, we can assume without loss of generality that $N+1-c \leq n+1$.

Case 1: $N+1-c=t_{i}$ and $t_{i}$ is a cut in $D(P, T)$. We claim that $t_{i}-1$ must be a cut as well. If $t_{i}=p_{i}$, then $p_{i-1}<t_{i}$, and we are done. Thus, we only need to consider the case that $t_{i}$ is an exceptional cut in $D(P, T)$.

If $t_{i}-1$ is not a visible cut, then $p_{i-1} \geq t_{i}$. In fact, if $t_{i}=n+1$, then $p_{i-1}=t_{i}$, since that is the only way the exceptional center cut can arise. On the other hand, if $t_{i} \neq n+1$, then, since $\#\left(\left[1, t_{i}\right] \cap T\right)=\#\left(\left[1, t_{i}\right] \cap P\right)+1$, row $i$ of $D(P, T)$ is the only row crossing the exceptional cut $t_{i}$. In this case too we must have $p_{i-1}=t_{i}$.

We therefore have $t_{i} \in[T] \cap[P]$. Furthermore, since row $i-1$ is the only row crossing from column $t_{i}-1$ to column $t_{i}$, we have $\#\left(\left[1, t_{i}-1\right] \cap T\right)=$ $\#\left(\left[1, t_{i}-1\right] \cap P\right)+1$. Thus $t_{i}-1$ is also an exceptional cut in $D(P, T)$.

Case 2: $N+1-c=p_{i}$ and $p_{i}-1$ is a cut in $D(P, T)$. We claim that $p_{i}$ must be a cut as well. As before, we can assume that $p_{i}-1$ is an exceptional cut in $D(P, T)$.

If $p_{i}$ is not a visible cut, then $t_{i+1} \leq p_{i}$. In fact, we must have $t_{i+1}=p_{i}$, since row $i$ is the only row crossing the exceptional cut $p_{i}-1$. 

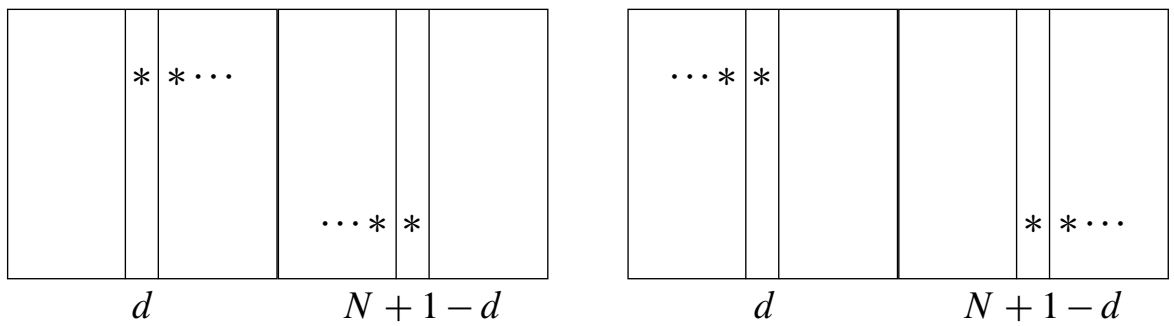

Figure 1. Conflicting lone stars in $D(P, T)$ in Proposition 4.12.

If $p_{i}=n+1$, then, since $t_{i+1}=p_{i}$, the diagram $D(P, T)$ has the exceptional center cut $n+1$, and we are done.

If $p_{i} \neq n+1$, then, since $t_{i+1}=p_{i}$, row $i+1$ must be the only row crossing from column $p_{i}$ to column $p_{i}+1$. Hence, $\#\left(\left[1, p_{i}\right] \cap T\right)=\#\left(\left[1, p_{i}\right] \cap P\right)+1$. Thus $p_{i}$ is also an exceptional cut in $D(P, T)$.

Given Schubert symbols $T \leq P$ in $\Omega(\mathrm{OG}(m, 2 n+2))$ such that $\mathfrak{t}(T) \neq \mathfrak{t}(P)$, we define a critical window in $D(P, T)$ to be an interval $[c+1, N-c]$ such that $c$ and $N-c$ are visible cuts in $D(P, T)$, and $[c+1, n+1] \subset[T] \cap[P]$.

Lemma 4.11. Given Schubert symbols $T<P$ in $\Omega(\mathrm{OG}(m, 2 n+2))$, we have $T \nprec P$ if and only if a critical window exists in $D(P, T)$.

Proof. If $T \nprec P$, then $\mathfrak{t}(P) \neq \mathfrak{t}(T)$ and there exists $c \in[1, n]$ such that $[c+1, n+1] \subset$ $[T] \cap[P]$ and $\#[1, c] \cap P=\#[1, c] \cap T$. By Corollary 4.8, both $c$ and $N-c$ are visible cuts in $D(P, T)$, and hence $[c+1, N-c]$ is a critical window. Conversely, if $D(P, T)$ has a critical window, then it is clear that $T \nprec P$.

The fact that $(P \cup T) \cap \mathscr{L}_{P, T}=\varnothing$ follows easily from the next proposition:

Proposition 4.12. Given $T<P$ in $\mathrm{OG}(m, 2 n+2), T \nprec P$ if and only if there exists an integer $d \in[1, N]$ such that $D(P, T)$ has lone stars in columns $d$ and $N+1-d$.

Proof. Suppose columns $d$ and $N+1-d$ of $D(P, T)$ both contain lone stars, and assume $d \leq n+1$. If $d=t_{i}$ for some $i$, then $N+1-d=p_{j}$ for some $j$, as shown in the left side of Figure 1. It follows that $t_{i} \neq p_{i}$, so $t_{i}$ must be an exceptional cut in $D(P, T)$. Thus $\left[t_{i}+1, n+1\right] \subset[T] \cap[P]$ and $\mathfrak{t}(T) \neq \mathfrak{t}(P)$. Furthermore, row $i$ must be the only row crossing from column $t_{i}$ to column $t_{i}+1$, and hence $p_{i-1}<t_{i}$, implying that $t_{i}-1$ is a visible cut. Therefore, $\left[t_{i}, p_{j}\right]$ is a critical window in $D(P, T)$. On the other hand, if $d=p_{i}$ for some $i$, then $N+1-d=t_{j}$ for some $j$, as shown in the right side of Figure 1 . In this case, $p_{i}-1$ must be an exceptional cut, $p_{i}$ must be a visible cut, and $\left[p_{i}+1, t_{i}-1\right]$ must be a critical window in $D(P, T)$. By Lemma 4.11, it follows that $T \not \subset P$. 
On the other hand, if $T \npreceq \npreceq P$, then by Lemma 4.11 there exists a critical window $[c+1, N-c]$ in $D(P, T)$. We claim that there exists a (possibly smaller) critical window of the form $\left[t_{i}, p_{j}\right]$ for some $i$ and $j$. To see why, note that if $[c+1, N-c]$ does not have the form $\left[t_{i}, p_{j}\right]$, then either $c+1=t_{i}=p_{i}$ or $N-c=t_{j}=p_{j}$. Either way, $[c+2, N-c-1]$ is a smaller critical window. However, this process of shrinking can't continue indefinitely. In particular, if $[c+1, N-c]=[n+1, n+2]$, then the fact that $[n+1, n+2]$ is a critical window implies that $t_{i}=n+1$ and $p_{i}=n+2$ for some $i$.

Finally, note that whenever $\left[t_{i}, p_{j}\right]$ is a critical window in $D(P, T)$ it must be the case that $t_{i}$ and $p_{j}-1$ are exceptional cuts. Thus $\left(i, t_{i}\right)$ and $\left(j, p_{j}\right)$ are lone stars and $t_{i}+p_{j}=N+1$, completing the proof.

Corollary 4.13. Given $T \preceq P$, we have $(P \cup T) \cap \mathscr{L}_{P, T}=\varnothing$.

Proof. Suppose $c \in(P \cup T) \cap \mathscr{L}_{P, T}$. Since $c$ is not a zero column, there exists a lone star in column $N+1-c$ of $D(P, T)$. By Proposition 4.10, $c$ and $c-1$ are both cuts in $D(P, T)$. But then column $c$ contains a lone star as well, since $c \in(P \cup T)$, contradicting Proposition 4.12.

4B. A complete intersection. The quadratic equation characterizing isotropic vectors in $\mathbb{C}^{2 n+2}$ is $f_{n+1}^{D}:=x_{1} x_{N}+\cdots+x_{n+1} x_{n+2}=0$. We once again let $2_{P, T}=([0, n] \cap \mathscr{C}) \cup\{n+1\}$. We let $Z_{P, T} \subset \mathbb{P}^{2 n+1}$ denote the subvariety cut out by the familiar polynomials $\left\{f_{c} \mid c \in \mathscr{2}_{P, T}\right\} \cup\left\{x_{c} \mid c \in \mathscr{L}_{P, T}\right\}$, where we let $f_{n+1}=f_{n+1}^{D}$.

It is not immediately obvious that $Z_{P, T}$ is a complete intersection in $\mathbb{P}^{N-1}$, or even that it is an irreducible subvariety. To prove these facts, we need the following lemma, which we prove in all three Lie types:

Lemma 4.14. Given Schubert symbols $T \preceq P$ for a Grassmannian $X$ of Lie-type $B, C$, or $D$, if $c-1$ and $c$ are both in $\mathscr{Q}_{P, T}$, then $c \in \mathscr{L}_{P, T}$ or $N+1-c \in \mathscr{L}_{P, T}$.

Proof. If we are working in type $B$, and $c=n+1$, then $n+1$ must be a zero column, and hence be in $\mathscr{L}_{P, T}$. Otherwise, we can assume that $c \leq\lfloor N / 2\rfloor$. If either $c$ or $N+1-c$ is a zero column in $D(P, T)$ then we are done, so assume neither column is empty.

Note that if $c-1$ is an exceptional cut, then $c \in[T] \cap[P]$. Otherwise, either $c-1$ or $N+1-c$ is a visible cut in $D(P, T)$, and hence $c \in T$ or $N+1-c \in P$ respectively, since neither $c$ nor $N+1-c$ is a zero column in $D(P, T)$.

In all of these cases $c \in[T] \cup[P]$. Therefore $(j, c)$ or $(j, N+1-c)$ is a lone star for some $j$. It follows that $N+1-c$ or $c$ is in $\mathscr{L}_{P, T}$.

We can now prove that $Z_{P, T}$ is a complete intersection in types $B, C$, and $D$. 
Proposition 4.15. Given Schubert symbols $T \preceq P$ for a Grassmannian $X$ of Lietype $B, C$, or $D$, the variety $Z_{P, T}$ is a complete intersection in $\mathbb{P}^{N-1}$ cut out by the following polynomials:

(a) $f_{d}-f_{c}=x_{c+1} x_{N-c}+\cdots+x_{d} x_{N+1-d}$, if $c$ and $d$ are consecutive elements of $\mathscr{2}_{P, T}$ such that $d-c \geq 2$.

(b) $x_{c}$, if $c \in \mathscr{L}_{P, T}$.

Proof. Let $I_{P, T} \subset \mathbb{C}\left[x_{1}, \ldots, x_{N}\right]$ be the ideal generated by the polynomials of types (a) and (b) mentioned in the statement of this proposition. Note that each of these polynomials is irreducible, and that by Corollary 2.7 and Proposition 4.10 no variable $x_{i}$ appears in multiple generators.

It follows that $\mathbb{C}\left[x_{1}, \ldots, x_{N}\right] / I_{P, T}$ is a tensor product over $\mathbb{C}$ of finitely many integral domains. Since $\mathbb{C}$ is algebraically closed, $\mathbb{C}\left[x_{1}, \ldots, x_{N}\right] / I_{P, T}$ must itself be an integral domain, by [Springer 2009, Lemma 1.5.2]. Hence $I_{P, T}$ is a prime ideal.

Let $I_{P, T}^{\prime}$ be the ideal generated by the polynomials used to define $Z_{P, T}$ : namely, $\left\{f_{c} \mid c \in \mathscr{Q}_{P, T}\right\} \cup\left\{x_{c} \mid c \in \mathscr{L}_{P, T}\right\}$. Note that each of the generators of $I_{P, T}$ is a linear combination of these defining polynomials, and is therefore contained in $I_{P, T}^{\prime}$.

On the other hand, note that whenever $d-1$ and $d$ are elements of $2_{P, T}$, the polynomial $f_{d}-f_{d-1}=x_{d} x_{N+1-d}$ is contained in $I_{P, T}$, by Lemma 4.14. Thus if $c<d$ are any consecutive elements of $\mathscr{2}_{P, T}$, then $f_{d}-f_{c} \in I_{P, T}$. Now, supposing $f_{c}$ is one of the quadratic polynomials defining $Z_{P, T}$, let $\left\{0=c_{0}<c_{1}<\cdots<c_{S}=c\right\}$ be the complete list of cuts between 0 and $c$. It follows that $f_{c}=f_{c}-f_{0}=$ $\left(f_{c_{s}}-f_{c_{s-1}}\right)+\left(f_{c_{s-1}}-f_{c_{s-2}}\right)+\cdots+\left(f_{c_{1}}-f_{c_{0}}\right) \in I_{P, T}$, and therefore that $I_{P, T}^{\prime} \subset I_{P, T}$.

We have shown that $I_{P, T}^{\prime}=I_{P, T}$, and hence that $Z_{P, T}$ is the zero set of a prime ideal. It follows that the polynomials used to define $I_{P, T}$ also cut out $Z_{P, T}$ as a complete intersection in $\mathbb{P}^{N-1}$.

\section{Result 2: $\psi\left(\pi^{-1}\left(Y_{P, T}\right)\right) \subset Z_{P, T}$}

Let $X:=\mathrm{OG}(m, 2 n+2)$ and $N:=2 n+2$. We would like to show that any vector lying in any subspace $\Sigma \in Y_{P, T}$ satisfies the equations defining $Z_{P, T}$. The equations involving exceptional cuts are the most difficult to verify, so we'll address them first.

Let $Y_{P, T}^{\circ}=X_{P}^{\circ}\left(E_{\bullet}\right) \cap X_{T^{\vee}}^{\circ}\left(E_{\bullet}^{\mathrm{op}}\right)$. It is a dense open subset of $Y_{P, T}$ (see [Richardson 1992]), so we can restrict our attention to $\psi\left(\pi^{-1}\left(Y_{P, T}^{\circ}\right)\right)$ :

Proposition 5.1. Consider Schubert symbols $T \preceq P$ for $\mathrm{OG}(m, 2 n+2)$, and suppose $c \in[1, n]$ is an exceptional cut in $D(P, T)$. Then $f_{c}(\boldsymbol{w})=0$ for all $\boldsymbol{w} \in \psi\left(\pi^{-1}\left(Y_{P, T}^{\circ}\right)\right)$. 
Proof. Since $c$ is exceptional, we know $[c+1, n+1] \subset[T] \cap[P]$, \#P $\cap[1, c]+1=$ $\# T \cap[1, c]$, and $\mathfrak{t}(P) \neq \mathfrak{t}(T)$.

Let $\ell=n+1-c$, and let $E^{(c)}=E_{N-c} / E_{c}$, which we identify with the span $\left\langle\boldsymbol{e}_{c+1}, \ldots, \boldsymbol{e}_{N-c}\right\rangle$. Finally, let $\alpha=\# P \cap[1, c]$.

Suppose $\Sigma$ is an element of $Y_{P, T}^{\circ}$. Since $\Sigma \in X_{P}^{\circ}$, we have $\operatorname{dim}\left(\Sigma \cap E_{c}\right)=\alpha$. Similarly, since $\Sigma \in X_{T^{\vee}}^{\circ}\left(E_{\bullet}^{\text {op }}\right)$, we have $\operatorname{dim}\left(\Sigma \cap E_{c}^{\text {op }}\right)=m-(\alpha+\ell+1)$. Furthermore, $\operatorname{dim}\left(\Sigma \cap E_{N-c}\right)=\alpha+\ell$ and $\operatorname{dim}\left(\Sigma \cap E_{N-c}^{\mathrm{op}}\right)=m-(\alpha+1)$. Finally, we know that

$$
\begin{aligned}
\operatorname{dim}\left(\Sigma \cap E^{(c)}\right) & =\operatorname{dim}\left(\Sigma \cap E_{N-c} \cap E_{N-c}^{\mathrm{op}}\right) \\
& \geq \operatorname{dim}\left(\Sigma \cap E_{N-c}\right)+\operatorname{dim}\left(\Sigma \cap E_{N-c}^{\mathrm{op}}\right)-m \\
& =(\alpha+\ell)+(m-(\alpha+1))-m \\
& =\ell-1 .
\end{aligned}
$$

Therefore we can choose vectors $\boldsymbol{u}_{1}$ through $\boldsymbol{u}_{m}$ spanning $\Sigma$ such that

$$
\begin{array}{lr}
\boldsymbol{u}_{i} \in E_{c}=\left\langle\boldsymbol{e}_{1}, \ldots, \boldsymbol{e}_{c}\right\rangle & \text { for } 1 \leq i \leq \alpha, \\
\boldsymbol{u}_{i} \in E^{(c)}=\left\langle\boldsymbol{e}_{c+1}, \ldots, \boldsymbol{e}_{N-c}\right\rangle & \text { for } \alpha+2 \leq i \leq \alpha+\ell, \\
\boldsymbol{u}_{i} \in E_{c}^{\mathrm{op}}=\left\langle\boldsymbol{e}_{N-c+1}, \ldots, \boldsymbol{e}_{N}\right\rangle & \text { for } \alpha+\ell+2 \leq i \leq m .
\end{array}
$$

In other words, $\Sigma$ can be represented as the rowspace of a matrix with the following shape (in the sense that all the entries outside the horizontal arrows are zero):

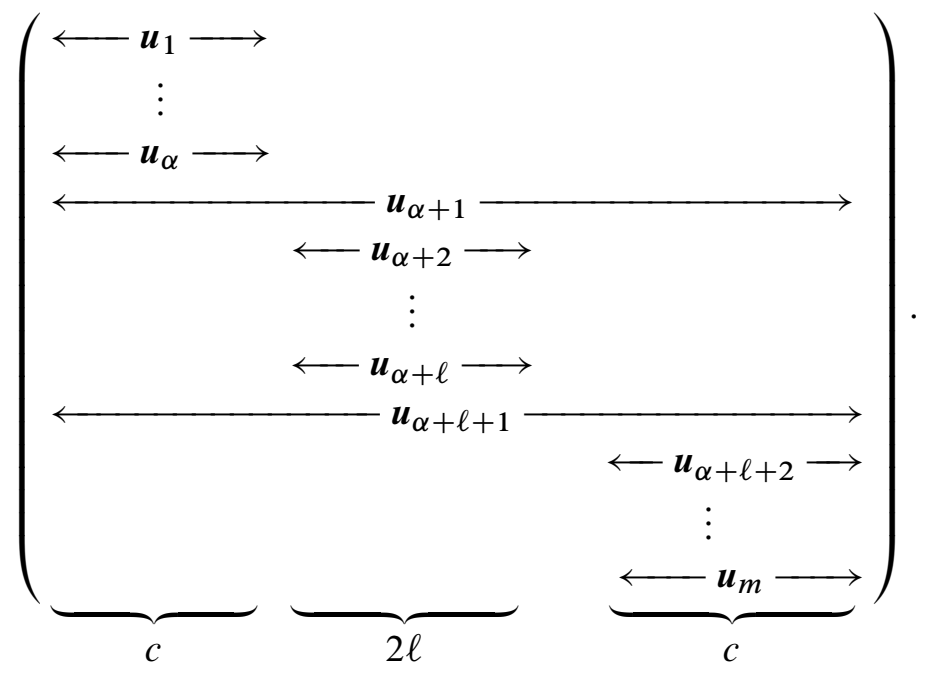


Furthermore, since $\operatorname{dim}\left(\Sigma \cap E_{N-c}\right)=\alpha+\ell$, we can assume without loss of generality that $\boldsymbol{u}_{\alpha+1} \in E_{N-c}$. The matrix with rowspace $\Sigma$ then has the following shape:

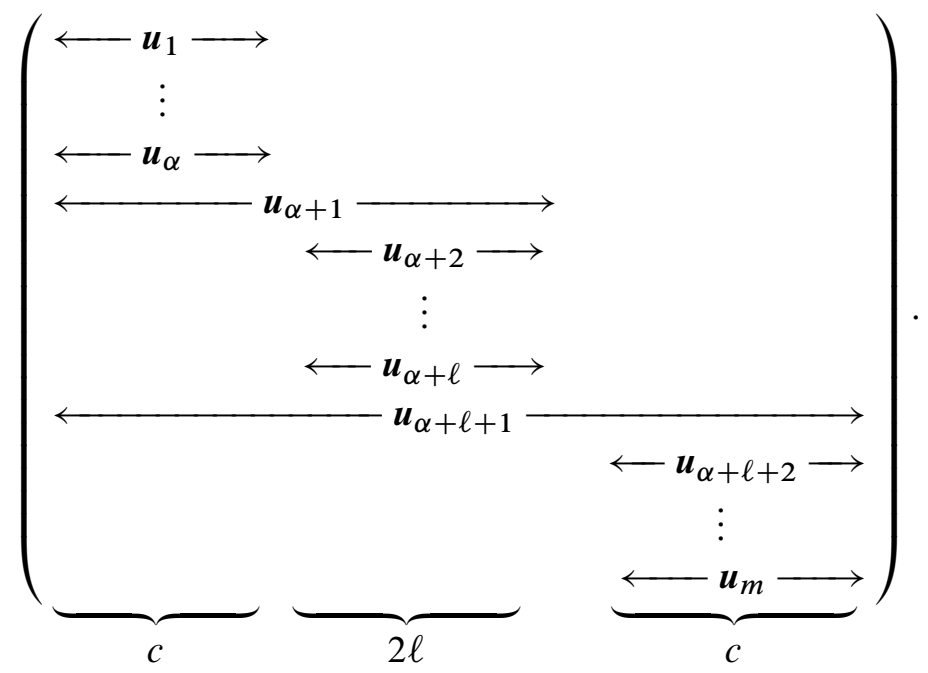

We shall now consider two cases, corresponding to whether or not $\boldsymbol{u}_{\alpha+1}$ is contained in $E_{N-c}^{\mathrm{op}}$ :

Case 1: $\boldsymbol{u}_{\alpha+1} \in E_{N-c}^{\mathrm{op}}$. The matrix with rowspace $\Sigma$ then has the following shape:

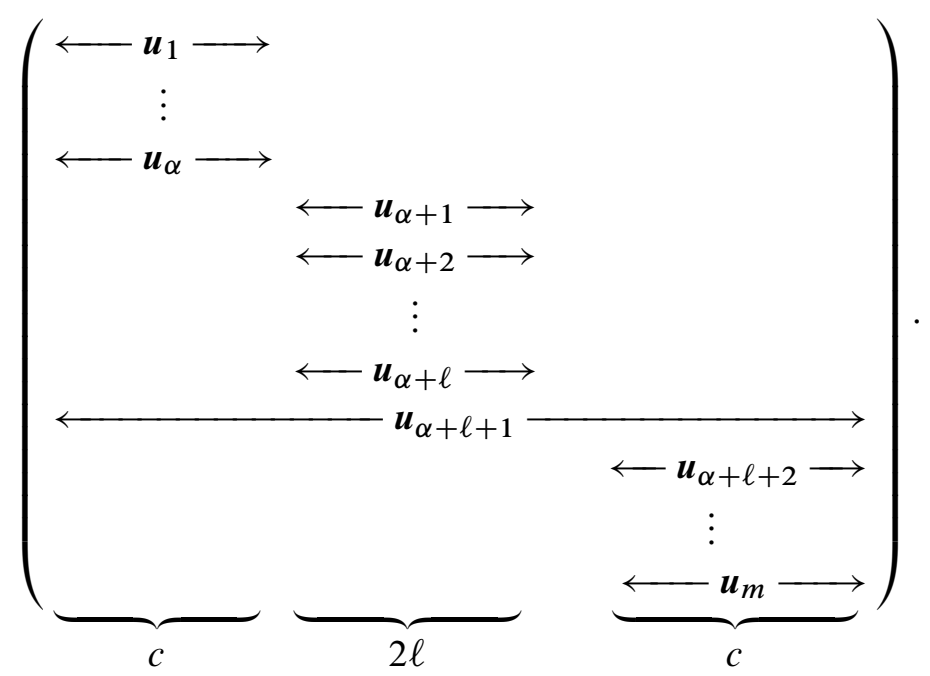

Note that for $1 \leq \beta \leq \alpha+\ell$, we have $u_{\beta, j}=0$ for any $N+1-c \leq j \leq N$. Thus for $1 \leq \beta \leq \alpha+\ell$ we have

$$
u_{\alpha+\ell+1,1} \cdot u_{\beta, N}+\cdots+u_{\alpha+\ell+1, c} \cdot u_{\beta, N+1-c}=0,
$$

where $u_{i, j}$ is the $j$-th coordinate of $\boldsymbol{u}_{i}$. 
Because $\Sigma$ is isotropic, we have $\omega\left(\boldsymbol{u}_{\alpha+\ell+1}, \boldsymbol{u}_{\beta}\right)=0$ for all $1 \leq \beta \leq m$. In particular, for $\alpha+\ell+2 \leq \beta \leq m$, we then have

$$
u_{\alpha+\ell+1,1} \cdot u_{\beta, N}+\cdots+u_{\alpha+\ell+1, c} \cdot u_{\beta, N+1-c}=0 .
$$

Finally, let $\boldsymbol{v}$ be the orthogonal projection of $\boldsymbol{u}_{\alpha+\ell+1}$ onto $E^{(c)}$. The span of $\boldsymbol{u}_{\alpha+1}, \ldots, \boldsymbol{u}_{\alpha+\ell+1}$ is a maximal isotropic subspace of $E^{(c)}$, so $\boldsymbol{v}$ must be contained in that span. In particular, $\boldsymbol{v}$ is itself an isotropic vector. Thus

$$
u_{\alpha+\ell+1,1} \cdot u_{\alpha+\ell+1, N}+\cdots+u_{\alpha+\ell+1, c} \cdot u_{\alpha+\ell+1, N+1-c}=0 .
$$

It follows that $f_{c}(\boldsymbol{w})=0$ for any vector $\boldsymbol{w}$ in $\Sigma$.

Case 2: $\boldsymbol{u}_{\alpha+1} \notin E_{N-c}^{\mathrm{op}}$. Since $\operatorname{dim}\left(\Sigma \cap E_{N-c}^{\mathrm{op}}\right)=m-(\alpha+1)$, we may assume $\boldsymbol{u}_{\alpha+\ell+1} \in E_{N-c}^{\mathrm{op}}$, after possibly adding a linear combination of $\boldsymbol{u}_{1}, \ldots, \boldsymbol{u}_{\alpha+1}$. Hence there exists a matrix with rowspace $\Sigma$ of the shape

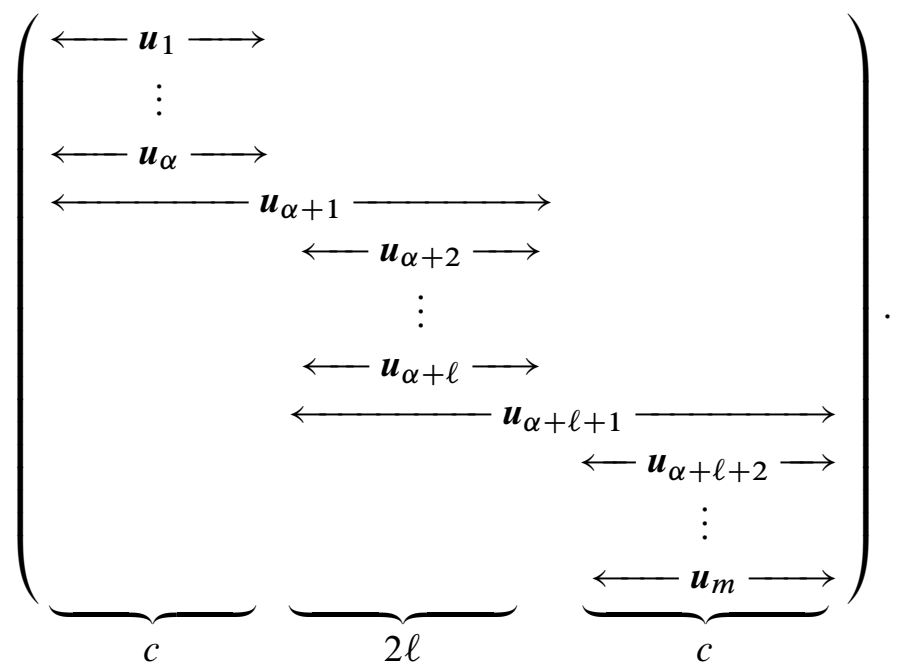

Let $\rho: \mathbb{C}^{N} \rightarrow \mathbb{C}^{N}$ be the orthogonal projection on to $E^{(c)}$. Notice that $\rho\left(\boldsymbol{u}_{i}\right)=\mathbf{0}$ for $i \leq \alpha$ and $i \geq \alpha+\ell+2$. Define $\boldsymbol{v}_{i}=\rho\left(\boldsymbol{u}_{\alpha+i}\right)$ for $i \in[1, \ell+1]$. Note that $\boldsymbol{v}_{i}=\boldsymbol{u}_{\alpha+i}$ for $i \in[2, \ell]$, but

$$
\begin{aligned}
\boldsymbol{v}_{1} & =\left(0, \ldots, 0, u_{\alpha+1, c+1}, \ldots, u_{\alpha+1, N-c}, 0, \ldots, 0\right), \\
\boldsymbol{v}_{\ell+1} & =\left(0, \ldots, 0, u_{\alpha+\ell+1, c+1}, \ldots, u_{\alpha+\ell+1, N-c}, 0, \ldots, 0\right),
\end{aligned}
$$

where $u_{i, j}$ is the $j$-th coordinate of $\boldsymbol{u}_{i}$.

Let

$$
\begin{aligned}
\Sigma^{\prime} & =\left\langle\boldsymbol{v}_{2}, \ldots, \boldsymbol{v}_{\ell}\right\rangle, \\
\Sigma_{1} & =\left\langle\boldsymbol{v}_{1}, \ldots, \boldsymbol{v}_{\ell}\right\rangle, \\
\Sigma_{2} & =\left\langle\boldsymbol{v}_{2}, \ldots, \boldsymbol{v}_{\ell+1}\right\rangle .
\end{aligned}
$$


Both $\Sigma_{1}$ and $\Sigma_{2}$ are elements of $\operatorname{OG}\left(\ell, E^{(c)}\right)$, and both contain $\Sigma^{\prime}$. It is a wellknown fact that there are exactly two isotropic $\ell$-planes containing a given isotropic $(\ell-1)$-plane [Fulton and Harris 1991, §23.3]. In particular, there are exactly two elements of $\operatorname{OG}\left(\ell, E^{(c)}\right)$ that contain $\Sigma^{\prime}$, so it remains to verify that $\Sigma_{1}$ and $\Sigma_{2}$ are indeed the same element. It is here that the types of $P$ and $T$ become relevant.

Namely, let

$$
\begin{aligned}
& P^{(c)}:=\{p-c: p \in P \cap[c+1, N-c]\}, \\
& T^{(c)}:=\{t-c: t \in T \cap[c+1, N-c]\}
\end{aligned}
$$

be Schubert symbols for $\mathrm{OG}\left(\ell, E^{(c)}\right) \cong \mathrm{OG}(\ell, 2 \ell)$. Note that whenever a subspace $\Lambda$ is contained in an intersection of Schubert cells $X_{R}^{\circ}$ and $X_{S^{\vee}}^{\circ}$ in $\operatorname{OG}(\ell, 2 \ell)$, it must be the case that $S \preceq R$ and hence that $\mathfrak{t}(\Lambda)=\mathfrak{t}(R)=\mathfrak{t}(S)$. In particular, $\Sigma_{1} \in$ $X_{P^{(c)}}^{\circ} \subset \mathrm{OG}(\ell, 2 \ell)$ by the definition of $X_{P^{(c)}}^{\circ}$, so it follows that $\mathfrak{t}\left(\Sigma_{1}\right)=\mathfrak{t}\left(P^{(c)}\right)$. Similarly, $\Sigma_{2} \in X_{T^{(c), \vee}}^{\circ} \subset \mathrm{OG}(\ell, 2 \ell)$ by Observation 3.4, and so $\mathfrak{t}\left(\Sigma_{2}\right)=\mathfrak{t}\left(T^{(c)}\right)$.

We claim that $\mathfrak{t}(P)=\mathfrak{t}(T)$ if and only if $\mathfrak{t}\left(P^{(c)}\right) \neq \mathfrak{t}\left(T^{(c)}\right)$. To see why, note that

$$
\begin{aligned}
& \#([1, n+1] \backslash P)=\#\left([1, \ell] \backslash P^{(c)}\right)+(c-\alpha), \\
& \#([1, n+1] \backslash T)=\#\left([1, \ell] \backslash T^{(c)}\right)+(c-(\alpha+1)) .
\end{aligned}
$$

Thus $\mathfrak{t}(P)+\mathfrak{t}(T) \equiv \mathfrak{t}\left(P^{(c)}\right)+\mathfrak{t}\left(T^{(c)}\right)+1(\bmod 2)$.

Since we know that $\mathfrak{t}(P) \neq \mathfrak{t}(T)$, we can conclude that $\Sigma_{1}=\Sigma_{2}$. It follows that $\boldsymbol{v}_{\ell+1} \in \Sigma_{1}$. But $\Sigma_{1}$ is isotropic, and therefore $\omega\left(\boldsymbol{v}_{1}, \boldsymbol{v}_{\ell+1}\right)=0$. Thus, for all $\boldsymbol{w} \in \Sigma$, the polynomial $x_{c+1} x_{N-c}+\cdots+x_{n+1} x_{n+2}$ vanishes, and hence $f_{c}(\boldsymbol{w})=0$.

Having addressed exceptional cuts, we can now prove a generalization of Lemma 2.8 for a Grassmannian $X$ of type $B, C$, or $D$ :

Proposition 5.2. Given Schubert symbols $T \preceq P$ in $\Omega(X)$, where $X$ is a Grassmannian of type $B, C$, or $D$, the projected Richardson variety $\psi\left(\pi^{-1}\left(Y_{P, T}\right)\right)$ is contained in $Z_{P, T}$.

Proof. Fix $\Sigma \in Y_{P, T}$ and $\boldsymbol{w}=\left(w_{1}, \ldots, w_{N}\right) \in \Sigma$. Suppose $c \in \mathscr{2}_{P, T}$. We will first show that $f_{c}(\boldsymbol{w})=0$.

If $c$ (or $N-c$ ) is a visible cut, then there exists $j \in[1, m]$ such that $p_{j} \leq c<t_{j+1}$ (or $p_{j} \leq N-c<t_{j+1}$ ).

Let $W_{1}=\left\langle\boldsymbol{e}_{1}, \ldots, \boldsymbol{e}_{p_{j}}\right\rangle$ and $W_{2}=\left\langle\boldsymbol{e}_{p_{j}+1}, \ldots, \boldsymbol{e}_{N}\right\rangle$. Since $\mathbb{C}^{N}=W_{1} \oplus W_{2}$, there exists a unique decomposition $\boldsymbol{w}=\boldsymbol{w}_{1}+\boldsymbol{w}_{2}$ with $\boldsymbol{w}_{i} \in W_{i}$. Namely, we have $\boldsymbol{w}_{1}=\left(w_{1}, \ldots, w_{p_{j}}, 0, \ldots, 0\right)$ and $\boldsymbol{w}_{2}=\left(0, \ldots, 0, w_{p_{j}+1}, \ldots, w_{N}\right)$. Note that, since $w_{i}=0$ for $p_{j}<i<t_{j+1}$, we have $\boldsymbol{w}_{2}=\left(0, \ldots, 0, w_{t_{j+1}}, \ldots, w_{N}\right)$, and hence $f_{c}(\boldsymbol{w})=\left(\boldsymbol{w}_{1}, \boldsymbol{w}_{2}\right)$. 
Since $\Sigma \in Y_{P, T}, \operatorname{dim}\left(\Sigma \cap W_{1}\right) \geq j$ and $\operatorname{dim}\left(\Sigma \cap W_{2}\right) \geq m-j$. We can therefore write $\Sigma=\left(\Sigma \cap W_{1}\right) \oplus\left(\Sigma \cap W_{2}\right)$, and decompose $\boldsymbol{w}$ as the sum of vectors in these subspaces. Since there is only one such decomposition, we must have $w_{1} \in \Sigma \cap W_{1}$ and $\boldsymbol{w}_{2} \in \Sigma \cap W_{2}$. Since $\Sigma$ isotropic, $f_{c}(\boldsymbol{w})=\left(\boldsymbol{w}_{1}, \boldsymbol{w}_{2}\right)=0$.

If $c=n+1$ (implying we are working in type $B$ or $D$ ), then $f_{c}=f_{n+1}$, the inherent quadratic equation, which vanishes on all isotropic vectors.

Lastly, if $c$ is exceptional, then $f_{c}(\boldsymbol{w})=0$ by Proposition 5.1.

We have shown that the projected Richardson variety $\psi\left(\pi^{-1}\left(Y_{P, T}\right)\right)$ lies in the zero set of the polynomial $f_{c}$ for any element $c \in 2_{P, T}$. In particular, it satisfies all the quadratic equations defining $Z_{P, T}$.

We must now show that $\psi\left(\pi^{-1}\left(Y_{P, T}\right)\right)$ satisfies the linear equation $x_{c}=0$ for any $c \in \mathscr{L}_{P, T}$. Suppose $c \in[1, N]$ is a zero column of $D(P, T)$, and let $\Sigma$ continue to denote an arbitrary element of $Y_{P, T}$. Let $j=\max \left\{i \in[1, m]: p_{i}<c\right\}$, and note that $t_{j+1}>c$. This time let $W_{1}=\left\langle\boldsymbol{e}_{1}, \ldots, \boldsymbol{e}_{c-1}\right\rangle$ and $W_{2}=\left\langle\boldsymbol{e}_{c+1}, \ldots, \boldsymbol{e}_{N}\right\rangle$. Thus, $\operatorname{dim}\left(\Sigma \cap W_{1}\right) \geq j$ and $\operatorname{dim}\left(\Sigma \cap W_{2}\right) \geq m-j$, so $\Sigma \subset W_{1} \oplus W_{2}$, which is the hyperplane defined by $x_{c}=0$.

Finally, suppose column $d \in[1, N]$ contains a lone star. In other words, $d=p_{j}$ or $d=t_{j}$ for some $j$, and $d-1$ and $d$ are both cuts in $D(P, T)$. Let $c=$ $\min (d, N+1-d)$, which is in $[1,\lceil N / 2\rceil]$. Both $c-1$ and $c$ are also cuts in $D(P, T)$, so the set $\psi\left(\pi^{-1}\left(Y_{P, T}\right)\right)$ must lie in the zero set of the polynomial $f_{c}-f_{c-1}=x_{c} x_{N+1-c}=x_{d} x_{N+1-d}$. However, since $\psi\left(\pi^{-1}\left(Y_{P, T}\right)\right)$ is irreducible (by [Richardson 1992]), it must lie in $x_{d}=0$ or $x_{N+1-d}=0$ (or both).

Consider any subspace $\Lambda$ in the dense subset $X_{P}^{\circ}\left(E_{\bullet}\right) \cap X_{T^{\vee}}^{\circ}\left(E_{\bullet}^{\mathrm{op}}\right) \subset Y_{P, T}$. Since $d \in P \cup T$, it is impossible that $x_{d}=0$ on all vectors in $\Lambda$. Therefore, the equation $x_{N+1-d}=0$ must be satisfied by $\psi\left(\pi^{-1}\left(Y_{P, T}\right)\right)$.

\section{Result 3: $Z_{P, T} \subset \psi\left(\pi^{-1}\left(Y_{P, T}\right)\right)$}

Let $X:=\mathrm{IG}_{\omega}\left(m, \mathbb{C}^{N}\right)$ be a Grassmannian of type $B, C$, or $D$. Given Schubert symbols $P$ and $T$ in $\Omega(X)$, we write $P \rightarrow T$ whenever

(i) $T \preceq P$;

(ii) $p_{i} \leq t_{i+1}$ for all $i$, unless $p_{i}=n+2$ and $t_{i+1}=n+1$ in type $D$; and

(iii) if $p_{i}=t_{i+1}$, then $p_{i}$ is not a cut in $D(P, T)$.

When $p_{i}>t_{i+1}$, we say $D(P, T)$ has a $2 \times 2$ square. Thus, the second condition says that $D(P, T)$ has no $2 \times 2$ squares, except that a single $2 \times 2$ square in the central columns is permitted in type $D$. When $p_{i}=t_{i+1}$ for some $i$ and $p_{i} \in \mathscr{C}_{P, T}$, then $\left(i+1, t_{i+1}\right)$ is a lone star in $D(P, T)$ (in fact, so is $\left(i, p_{i}\right)$ ), and hence $N+1-p_{i} \in \mathscr{L}_{P, T}$. If we are working in type $B$ or $C$, then $N+1-p_{i}$ must be a zero column, which yields the equivalent definition of $P \rightarrow T$ given in [Buch et al. 2009]. 
If we are working in type $D$, then there are other possibilities involving exceptional cuts. For example, if $p_{i}=t_{i+1} \in\{n+1, n+2\}$, then $n+1$ will be an exceptional cut, causing both $\left(i, p_{i}\right)$ and $\left(i+1, t_{i+1}\right)$ to be lone stars. It follows that, when $P \rightarrow T$, the diagram $D(P, T)$ cannot have exactly three stars in the central columns $n+1$ and $n+2$, which yields the alternative type- $D$ definition of $P \rightarrow T$ given in [Buch et al. 2009, §5.2].

The relation $P \rightarrow T$ is important because it characterizes precisely when the map $\psi: \pi^{-1}\left(Y_{P, T}\right) \rightarrow Z_{P, T}$ is a birational isomorphism. In particular, [Buch et al. 2009, Proposition 5.1] says the following:

Proposition 6.1. Given a Grassmannian $X$ of Lie-type $B, C$, or $D$, the map $\psi: \pi^{-1}\left(Y_{P, T}\right) \rightarrow Z_{P, T}$ is a birational isomorphism if and only if the Schubert symbols $P$ and $T$ satisfy the relation $P \rightarrow T$.

A more detailed proof of Proposition 6.1 can be found in [Ravikumar 2013, §8]. In this section, we prove the following proposition:

Proposition 6.2. Given Schubert symbols $T \preceq P$ for a Grassmannian $X$ of type B, $C$, or D, there exists a Schubert symbol $\widetilde{P}$ such that
(1) $T \preceq \widetilde{P} \preceq P$,
(2) $Z_{\widetilde{P}, T}=Z_{P, T}$, and
(3) $\widetilde{P} \rightarrow T$.

We prove Proposition 6.2 by explicitly constructing the Schubert symbol $\widetilde{P}$. An immediate consequence is that, for any $T \preceq P$ in $\Omega(X)$, the Richardson variety $Y_{P, T}$ must contain a smaller Richardson variety $Y_{\widetilde{P}, T}$ such that $\psi: \pi^{-1}\left(Y_{\widetilde{P}, T}\right) \rightarrow$ $Z_{\widetilde{P}, T}=Z_{P, T}$ is a birational isomorphism (by Proposition 6.1). It follows that $Z_{P, T} \subset \psi\left(\pi^{-1}\left(Y_{P, T}\right)\right)$. By combining this observation with Proposition 5.2, which states that $Z_{P, T} \supset \psi\left(\pi^{-1}\left(Y_{P, T}\right)\right)$, we have a proof of Theorem 1.1. We mention that Proposition 6.2 is not the only way to prove that $Z_{P, T} \subset \psi\left(\pi^{-1}\left(Y_{P, T}\right)\right)$. For example, instead of "lowering" $P$ we could "raise" $T$. In fact, the construction we give for $\widetilde{P}$ does precisely that when carried out on the rotated diagram $D(\bar{T}, \bar{P})$.

6A. Constructing a "smaller" Schubert symbol $\boldsymbol{P}^{\prime}$. Given Schubert symbols $T \preceq P$, define the set $P^{\prime}=\left\{p_{1}^{\prime}, \ldots, p_{m}^{\prime}\right\}$ as follows:

If $p_{i}<t_{i+1}$, then

$$
p_{i}^{\prime}=p_{i}
$$

On the other hand, if $p_{i} \geq t_{i+1}$ and $t_{i+1}-1 \notin \mathscr{C}_{P, T}$, then

$$
p_{i}^{\prime}=t_{i+1} \text {. }
$$


Finally, if $p_{i} \geq t_{i+1}$ and $t_{i+1}-1 \in \mathscr{C}_{P, T}$, then

$$
p_{i}^{\prime}=\max \left\{c \in\left[t_{i}, t_{i+1}-1\right] \mid c \notin \mathscr{L}_{P, T}\right\} .
$$

Note that since $t_{i}$ cannot be in $\mathscr{L}_{P, T}$, the set $\left\{c \in\left[t_{i}, t_{i+1}-1\right] \mid c \notin \mathscr{L}_{P, T}\right\}$ is nonempty. Thus $p_{i}^{\prime}$ is well-defined.

The next property of $P^{\prime}$ follows from its construction and Corollary 4.13:

Observation 6.3. For any $1 \leq i \leq m, p_{i}^{\prime} \notin \mathscr{L}_{\boldsymbol{P}, T}$.

Proof. If $p_{i}^{\prime}$ is defined by case $(\diamond)$ or case (\$), then $p_{i}^{\prime} \in P \cup T$, so by Corollary 4.13 it is not in $\mathscr{L}_{P, T}$. If it is defined by case $(\mathcal{O})$, then by its construction it cannot be in $\mathscr{L}_{P, T}$.

We also have the next observation, which follows directly from the construction of $P^{\prime}$ :

Observation 6.4. If $p_{i}^{\prime}$ is produced by case $(\diamond)$ or case $(\varnothing)$ of the previous construction, then $\left[p_{i}^{\prime}, t_{i+1}-1\right] \subset \mathscr{C}_{P, T}$. Furthermore, if $p_{i}^{\prime}<t_{i+1}-1$, then $\left[p_{i}^{\prime}+1, t_{i+1}-1\right] \subset \mathscr{L}_{P, T}$.

Lemma 6.5. $P^{\prime}$ is a Schubert symbol.

Proof. We will first show that $p_{i}^{\prime}<p_{i+1}^{\prime}$ for $1 \leq i \leq m-1$. By our construction of $P^{\prime}$, we have $p_{i}^{\prime} \leq t_{i+1} \leq p_{i+1}^{\prime}$ for each $i$. Thus we only need to consider the case that $p_{i+1}^{\prime}=t_{i+1}$. If $p_{i+1}^{\prime}=t_{i+1}$, then $p_{i+1}^{\prime}$ follows case $(\diamond)$ or case $(\nabla)$, so, by Observation 6.4, $t_{i+1} \in \mathscr{C}_{P, T}$. But then $\left(i+1, t_{i+1}\right)$ is a lone star in $D(P, T)$, so $t_{i+1}-1 \in \mathscr{C}_{P, T}$ as well (by Corollary 2.7). Thus $p_{i}^{\prime}$ follows case $(\diamond)$ or case $(\nabla)$, and we have $p_{i}^{\prime}<t_{i+1}$. It follows that $p_{i}^{\prime}<p_{i+1}^{\prime}$ for all $1 \leq i \leq m-1$, and in particular that $P^{\prime}$ consists of $m$ distinct integers.

We still have to check that $P^{\prime}$ satisfies the isotropic condition. Suppose on the contrary that $p_{i}^{\prime}+p_{j}^{\prime}=N+1$ for some $i$ and $j$ in $[1, m]$. By Observation 6.4, if $p_{i}^{\prime}$ is not a cut in $D(P, T)$, then $p_{i}^{\prime}=t_{i+1}$. Similarly, if $p_{j}^{\prime}$ is not a cut in $D(P, T)$, then $p_{j}^{\prime}=t_{j+1}$.

We therefore have three possible cases to consider, each of which results in a contradiction:

Case 1: Both $p_{i}^{\prime}$ and $p_{j}^{\prime}$ are cuts in $D(P, T)$. In this case $N-p_{j}^{\prime}=p_{i}^{\prime}-1$ is also a cut, and by Lemma 4.14 either $p_{i}^{\prime}$ or $p_{j}^{\prime}$ is in $\mathscr{L}_{P, T}$. But neither $p_{i}^{\prime}$ nor $p_{j}^{\prime}$ can be in $\mathscr{L}_{P, T}$, by Observation 6.3.

Case 2: Exactly one of $\left\{p_{i}^{\prime}, p_{j}^{\prime}\right\}$ is a cut in $D(P, T)$. We will assume without loss of generality that $p_{j}^{\prime}$ is a cut in $D(P, T)$. We then have $p_{i}^{\prime}-1 \in \mathscr{C}_{P, T}$, as in the previous case, implying that $p_{i}^{\prime}$ cannot follow case (\$). However, since $p_{i}^{\prime} \notin_{\mathscr{C}_{P}, T}$, Observation 6.4 implies that $p_{i}^{\prime}$ does follow case (\$), a contradiction. 
Case 3: Neither $p_{i}^{\prime}$ nor $p_{j}^{\prime}$ is a cut in $D(P, T)$. In this case, $p_{i}^{\prime}=t_{i+1}$ and $p_{j}^{\prime}=t_{j+1}$. But $t_{i+1}+t_{j+1} \neq N+1$, since $T$ is a Schubert symbol, so once again we arrive at a contradiction.

6B. Types $B$ and $C$. If $X$ is a Grassmannian of type $B$ or $C$, we set $\widetilde{P}:=P^{\prime}$ in order to prove Proposition 6.2. The type- $D$ case of Proposition 6.2 will be addressed in Section 6C.

Since $T \preceq P$ if and only if $T \leq P$, it is clear by our construction that $t_{i} \leq \tilde{p}_{i} \leq p_{i}$ for $1 \leq i \leq m$, and hence $T \preceq \widetilde{P} \preceq P$. Thus $\widetilde{P}$ satisfies Proposition 6.2(1). We will need the following lemma to verify the remaining conditions:

Lemma 6.6. The diagrams $D(P, T)$ and $D(\widetilde{P}, T)$ have the same cuts.

Proof. Given an integer $c \in[1, N]$, if $p_{i} \leq c<t_{i+1}$, then $\tilde{p}_{i}=p_{i} \leq c<t_{i+1}$. Therefore $\mathscr{C}_{P, T} \subset \mathscr{C}_{\tilde{P}, T}$. On the other hand, if $\tilde{p}_{i}<t_{i+1}$, then $\tilde{p}_{i}$ is defined by $(\diamond)$ or $(\nabla)$. We then have $\left[\tilde{p}_{i}, t_{i+1}-1\right] \subset \mathscr{C}_{P, T}$, by Observation 6.4. Therefore $\mathscr{C}_{P, T} \supset \mathscr{C}_{\widetilde{P}, T}$

Proof of Proposition 6.2. We show that $\widetilde{P}$ satisfies conditions (2) and (3).

$\widetilde{P}$ satisfies (2) $\left(Z_{P, T}=Z_{\widetilde{P}, T}\right):$ By Lemma 6.6, the diagrams $D(P, T)$ and $D(\widetilde{P}, T)$ have the same cuts. We still have to prove that $\mathscr{L}_{P, T}=\mathscr{L}_{\widetilde{P}, T}$. Suppose $c \in \mathscr{L}_{P, T}$. Then, by Proposition 2.6, either $p_{i}=t_{i}=N+1-c$ for some $i$ or $c$ is a zero column of $D(P, T)$. If $p_{i}=t_{i}=N+1-c$ for some $i$, then $p_{i}<t_{i+1}$, so $\tilde{p}_{i}$ must follow case $(\diamond)$ of the construction. Thus $\tilde{p}_{i}=t_{i}=N+1-c$, so $c \in \mathscr{L}_{\widetilde{P}, T}$. On the other hand, if $c$ is a zero column in $D(P, T)$, then $p_{i}<c<t_{i+1}$ for some $i$. Once again we have $\tilde{p}_{i}=p_{i}$, so $c$ is a zero column in $D(\widetilde{P}, T)$, and therefore an element of $\mathscr{L}_{\widetilde{P}, T}$.

Now suppose $c \in \mathscr{L}_{\widetilde{P}, T}$. If $\tilde{p}_{i}<c<t_{i+1}$ for some $i$, then $\tilde{p}_{i}$ satisfies $(\diamond)$ or $(\mathcal{Q})$. Either way, we must have $c \in \mathscr{L}_{P, T}$. On the other hand, if $(i, N+1-c)$ is a lone star in $D(\widetilde{P}, T)$ for some $i$, then $\tilde{p}_{i}=t_{i}=N+1-c$. Thus, $\tilde{p}_{i} \in \mathscr{C}_{\widetilde{P}, T}=\mathscr{C}_{P, T}$. In this case $\left(i, t_{i}\right)$ is a lone star in $D(P, T)$, and $c \in \mathscr{L}_{P, T}$.

$\widetilde{P}$ satisfies (3) $(\tilde{P} \rightarrow T)$ : Since $\tilde{p}_{i} \leq t_{i+1}$ for $1 \leq i \leq m-1$, the diagram $D(\widetilde{P}, T)$ has no $2 \times 2$ squares. If $\tilde{p}_{i}=t_{i+1}$ for some $i$, then $t_{i+1}-1$ is not a cut in $D(P, T)$. By Lemma 6.6 it is also not a cut in $D(\widetilde{P}, T)$, so column $N+1-\tilde{p}_{i}$ cannot be a zero column in $D(\widetilde{P}, T)$.

6C. Type $D$. Let $X:=\mathrm{OG}(m, 2 n+2)$ be a type- $D$ Grassmannian, let $N:=2 n+2$, and let $T \preceq P$ be Schubert symbols in $\Omega(X)$. Unfortunately the Schubert symbol $P^{\prime}$ constructed in Section 6A fails to satisfy the conditions of Proposition 6.2, as the following example illustrates: 
Example 6.7. Consider $\mathrm{OG}(2,6)$, and let $T=\{1,3\}$ and $P=\{5,6\}$. The projected Richardson variety $Z_{P, T}$ is the quadric hypersurface of $\mathbb{P}^{5}$ consisting of all isotropic lines in $\mathbb{C}^{6}$. Since $P^{\prime}=\{3,6\}$ and the variety $Z_{P^{\prime}, T}$ satisfies the additional linear equation $x_{4}=0$, we have $Z_{P, T} \neq Z_{P^{\prime}, T}$. Furthermore, $P^{\prime} \not \rightarrow T$, since $p_{1}=t_{2}=3$ is a cut in $D\left(P^{\prime}, T\right)$ - the exceptional center cut

$$
\left(\begin{array}{llllll}
* & * & * & 0 & 0 & 0 \\
0 & 0 & * & * & * & *
\end{array}\right)
$$

We therefore define the set $\widetilde{P}=\left\{\tilde{p}_{1}, \ldots, \tilde{p}_{m}\right\}$ as follows:

If $p_{i}<t_{i+1}$, then

$$
\tilde{p}_{i}=p_{i}
$$

On the other hand, if $p_{i} \geq t_{i+1}$ and $t_{i+1}-1 \notin \mathscr{C}$, then

$$
\tilde{p}_{i}= \begin{cases}t_{i+1} & \text { if } t_{i+1} \notin\{n+1, n+2\}, \\ N+1-t_{i+1} & \text { if } t_{i+1} \in\{n+1, n+2\} .\end{cases}
$$

Finally, if $p_{i} \geq t_{i+1}$ and $t_{i+1}-1 \in \mathscr{C}$, then

$$
\tilde{p}_{i}=\max \left\{c \in\left[t_{i}, t_{i+1}-1\right] \mid c \notin \mathscr{L}\right\} .
$$

Note that since $t_{i}$ cannot be in $\mathscr{L}$, the set $\left\{c \in\left[t_{i}, t_{i+1}-1\right] \mid c \notin \mathscr{L}\right\}$ is nonempty. Thus $\tilde{p}_{i}$ is well-defined.

Recall that $\iota$ is the permutation of $\{1, \ldots, 2 n+2\}$ that interchanges $n+1$ and $n+2$ and leaves all other numbers fixed. We make the following observation:

Observation 6.8. $\widetilde{P}$ is equal to $\iota P^{\prime}$ or $P^{\prime}$. Moreover, $\widetilde{P}=\iota P^{\prime}$ if and only if there exists an element $\tilde{p}_{i}$ defined by case $(\dagger)$ and $t_{i+1} \in\{n+1, n+2\}$.

Given a type- $D$ Schubert symbol $R$, the set $\iota R$ is also a Schubert symbol, since the isotropic condition is preserved. We therefore have the following corollary:

Corollary 6.9. $\widetilde{P}$ is a Schubert symbol.

The following observations are exact restatements of Observations 6.3 and 6.4 for type $D$. We give a brief proof of the first, whereas the second follows directly from the construction of $\widetilde{P}$.

Observation 6.10. For any $1 \leq i \leq m, \tilde{p}_{i} \notin \mathscr{L}_{P, T}$.

Proof. If $\tilde{p}_{i}$ is defined by case $(\star)$, or if it is defined by case $(\dagger)$ and $t_{i+1} \notin$ $\{n+1, n+2\}$, then $\tilde{p}_{i} \in P \cup T$. By Corollary 4.13 , it is not in $\mathscr{L}_{P, T}$. If it is defined by case $(\$)$, then by its construction it cannot be in $\mathscr{L}_{P, T}$.

Finally, suppose $\tilde{p}_{i}$ is defined by case $(\dagger)$ and $t_{i+1} \in\{n+1, n+2\}$. If $\tilde{p}_{i}=$ $N+1-t_{i+1} \in \mathscr{L}_{P, T}$, then $t_{i+1}-1 \in \mathscr{C}_{P, T}$, contradicting the assumption that $\tilde{p}_{i}$ is defined by case $(\dagger)$. 


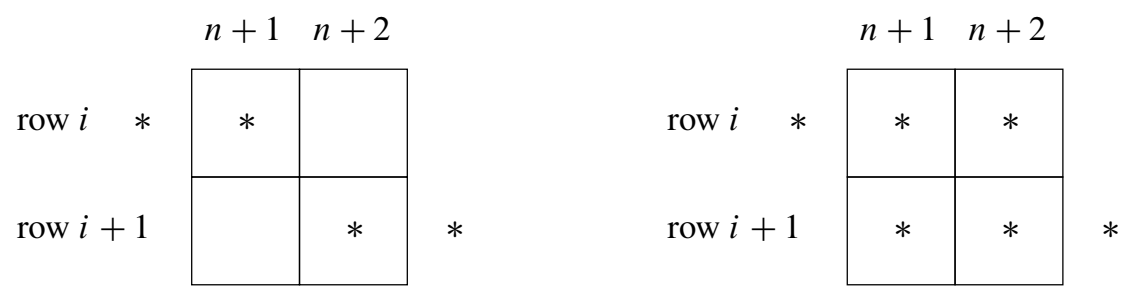

Figure 2. Center columns of $D(\widetilde{P}, T)$ in Lemma 6.12 .

Observation 6.11. If $\tilde{p}_{i}$ is produced by case ( $\star$ ) or case (\$) of the previous construction, then $\left[\tilde{p}_{i}, t_{i+1}-1\right] \subset \mathscr{C}_{P, T}$. Furthermore, if $\tilde{p}_{i}<t_{i+1}-1$, then $\left[\tilde{p}_{i}+1, t_{i+1}-1\right] \subset \mathscr{L}_{\boldsymbol{P}, T}$.

We will now proceed to prove that $T \preceq P, \widetilde{P} \rightarrow T, Z_{P, T}=Z_{\widetilde{P}, T}$, and $\widetilde{P} \preceq P$, in that order. We begin with an important lemma:

Lemma 6.12. If $n+1 \in[T] \cap[\widetilde{P}]$, then $\mathfrak{t}(T)=\mathfrak{t}(\widetilde{P})$.

Proof. Suppose $\tilde{p}_{i} \in\{n+1, n+2\}$ for some $i$ and $t_{j} \in\{n+1, n+2\}$ for some $j$.

Since $\tilde{p}_{k} \geq t_{k}>n+2$ for all $k>j$, we have $i \leq j$. Furthermore, $\tilde{p}_{k-1} \leq t_{k}<n+1$ for all $k<j$, so $i \geq j-1$. Thus we either have $i=j$ or $i+1=j$.

Case 1: $i=j$. We claim that $t_{i}=\tilde{p}_{i} \in\{n+1, n+2\}$. Suppose on the contrary that $t_{i}=n+1$ and $\tilde{p}_{i}=n+2$. Since $t_{i+1} \neq n+1, \tilde{p}_{i}$ is not defined by $(\dagger)$. Therefore, by Observation 6.11, $\tilde{p}_{i} \in \mathscr{C}_{P, T}$. It follows that both $n$ and $n+2$ are in $\mathscr{2}_{P, T}$, so, by Lemma 4.14, either $n+1$ or $n+2$ is in $\mathscr{L}_{P, T}$. But neither $t_{i}$ nor $\tilde{p}_{i}$ can be in $\mathscr{L}_{P, T}$, by Corollary 4.13 and Observation 6.10. To avoid this contradiction, we must have $t_{i}=\tilde{p}_{i} \in\{n+1, n+2\}$. Thus, $n+1$ is a visible cut in $D(\tilde{P}, T)$, and $\#([1, n+1] \cap T)=\#([1, n+1] \cap \widetilde{P})$. In other words, $\mathfrak{t}(T)=\mathfrak{t}(\widetilde{P})$.

Case 2: $i+1=j$. If $\tilde{p}_{i}=t_{i+1}$, then $\tilde{p}_{i}$ is defined by $(\dagger)$ and $t_{i+1} \notin\{n+1, n+2\}$. Hence $\tilde{p}_{i} \notin\{n+1, n+2\}$, a contradiction. It follows that $\tilde{p}_{i} \neq t_{i+1}$. If $\tilde{p}_{i}=n+1$ and $t_{i+1}=n+2$, then $n+1$ is a visible cut in $D(\widetilde{P}, T)$, as seen on the left side of Figure 2. On the other hand, if $\tilde{p}_{i}=n+2$ and $t_{i+1}=n+1$, as seen on the right side, then $\#([1, n+1] \cap T)=\#([1, n+1] \cap \widetilde{P})+2$, so once again $\mathfrak{t}(T)=\mathfrak{t}(\widetilde{P})$.

Lemma 6.12 says that $\{\mathfrak{t}(\widetilde{P}), \mathfrak{t}(T)\} \neq\{0,1\}$. Since we also know that $T \leq \widetilde{P}$ by construction, we have the following immediate corollaries:

Corollary 6.13. $T \preceq \widetilde{P}$.

Proof. The condition that $\{\mathfrak{t}(\widetilde{P}), \mathfrak{t}(T)\}=\{0,1\}$ is necessary for $T \nprec \widetilde{P}$.

Corollary 6.13 says that $\widetilde{P}$ satisfies the first half of condition (1) of Proposition 6.2. We prove the second half in Proposition 6.21.

Corollary 6.14. The diagram $D(\widetilde{P}, T)$ has no exceptional cuts, except possibly the center cut $n+1$. 
Proof. The condition that $\{\mathfrak{t}(\widetilde{P}), \mathfrak{t}(T)\}=\{0,1\}$ is necessary for the existence of an exceptional cut other than the center cut.

In fact, any noncentral exceptional cut in $D(P, T)$ becomes a visible cut in $D(\widetilde{P}, T)$. However, we will only need this fact in the special case that the exceptional cut is less than $n+1$ :

Lemma 6.15. If $1 \leq c \leq n$ is an exceptional cut in $D(P, T)$, then $c$ is a visible cut in $D(\widetilde{P}, T)$.

Proof. Since $c$ is an exceptional cut in $D(P, T)$, we have that $\#([1, c] \cap T)=$ $\#([1, c] \cap P)+1$. In other words, there exists exactly one integer $i \in[1, m]$ such that $t_{i} \leq c<p_{i}$.

Since row $i$ is the only row crossing from column $c$ to column $c+1$, we must have $c+1 \leq t_{i+1}$. Furthermore, since $n+1 \in[T]$, we must have $t_{i+1} \leq n+2$. We will show that $\tilde{p}_{i} \leq c$, and hence that $c$ is a visible cut in $D(\tilde{P}, T)$. We divide the rest of our argument into two cases:

Case 1: $c+1=t_{i+1}$. Since $c \in \mathscr{C}_{P, T}, \tilde{p}_{i}$ is defined by (\$), it follows that $\tilde{p}_{i} \leq c$.

Case 2: $c+1<t_{i+1} \leq n+2$. We claim that $p_{i} \geq t_{i+1}$. To see why, suppose for the sake of contradiction that $p_{i}<t_{i+1}$. Then $p_{i}$ is a visible cut in $D(P, T)$, and $\#\left(\left[1, p_{i}\right] \cap T\right)=\#\left(\left[1, p_{i}\right] \cap P\right)$. If $p_{i}<n+1$, then since $\left[p_{i}+1, n+1\right] \subset[P] \cap[T]$ and $\mathfrak{t}(T) \neq \mathfrak{t}(P)$ (which follow from the fact that $c$ is an exceptional cut), we have $T \nprec P$, a contradiction. If $p_{i}=n+1$, then $\#([1, n+1] \cap T)=\#([1, n+1] \cap P)$, so $\mathfrak{t}(P)=\mathfrak{t}(T)$, contradicting the assumption that $c$ is an exceptional cut. Finally, if $p_{i}>n+1$, then $t_{i+1}>n+2$, contradicting the assumption that $n+1 \in[T]$. It follows that $p_{i} \geq t_{i+1}$, as claimed.

Now note that any $d \in\left[c+1, t_{i+1}-1\right]$ must be an exceptional cut in $D(P, T)$, since $\#([1, d] \cap T)=\#([1, d] \cap P)+1$ (in particular row $i$ is the only row crossing from column $d$ to column $d+1)$. Furthermore, for each $d \in\left[c+1, t_{i+1}-1\right]$, we have $N+1-d \in T$, since $d \notin T$ and $[c+1, n+1] \in[T]$. It follows that column $N+1-d$ contains a lone star in $D(P, T)$, and hence that $d \in \mathscr{L}_{P, T}$. Since $\left[c+1, t_{i+1}-1\right] \subset \mathscr{L}_{P, T}$, it follows that $\tilde{p}_{i}$ is defined by (†) and that $\tilde{p}_{i} \leq c$.

The following proposition will also be needed:

Proposition 6.16. $\mathscr{C}_{P, T} \cup\{n+1\}=\mathscr{C}_{\widetilde{P}, T} \cup\{n+1\}$.

Proof. By Corollary 6.14, there are no exceptional cuts in $D(\widetilde{P}, T)$. If $c$ is a visible cut in $D(\tilde{P}, T)$, then $\tilde{p}_{i} \leq c<t_{i+1}$ for some $1 \leq i \leq m-1$. If $\tilde{p}_{i}$ is defined, by $(\star)$ or $(\ddagger)$, then, by Observation $6.11, c \in \mathscr{C}_{P, T}$. Otherwise $\tilde{p}_{i}$ is defined by $(\dagger)$ and $\tilde{p}_{i}=c=n+1$. Since all visible cuts in $\mathscr{C}_{\widetilde{P}, T}$ are contained in $\mathscr{C}_{P, T} \cup\{n+1\}$, the same is true for apparent cuts. It follows that $\mathscr{C}_{\widetilde{P}, T} \subset \mathscr{C}_{P, T} \cup\{n+1\}$. 
On the other hand, if $p_{i}<t_{i+1}$ then $\tilde{p}_{i}=p_{i}<t_{i+1}$. Thus any visible cut $c \in \mathscr{C}_{P, T}$ is also contained in $\mathscr{C}_{\widetilde{P}, T}$. If $c<n+1$ is an exceptional cut in $D(P, T)$, then, by Lemma $6.15, c \in \mathscr{C}_{\widetilde{P}, T}$. It follows that $\mathscr{C}_{P, T} \subset \mathscr{C}_{\widetilde{P}, T} \cup\{n+1\}$.

We can now prove that $\widetilde{P}$ satisfies condition (3) of Proposition 6.2.

\section{Proposition 6.17. $\widetilde{P} \rightarrow T$.}

Proof. By Corollary 6.13, $T \preceq \widetilde{P}$. The construction of $\widetilde{P}$ ensures that $\tilde{p}_{i}$ is never greater than $t_{i+1}$ except possibly when $t_{i+1}=n+1$ and $\tilde{p}_{i}=n+2$. Furthermore, if $\tilde{p}_{i}=t_{i+1}$, then $t_{i+1}-1$ is not a cut in $D(P, T)$. Therefore $t_{i+1}$ cannot be a cut in $D(P, T)$ either, since that would make $\left(i+1, t_{i+1}\right)$ a lone star in $D(P, T)$ and hence make $t_{i+1}-1$ a cut in $D(P, T)$, by Proposition 4.10. It follows that $t_{i+1}$ is not a cut in $D(\widetilde{P}, T)$, by Proposition 6.16 .

We will now show that $Z_{P, T}=Z_{\widetilde{P}, T}$ by examining the diagrams $D(P, T)$ and $D(\widetilde{P}, T)$. Recall that the quadratic equations defining $Z_{P, T}$ are entirely determined by $\mathscr{C}_{P, T}$, the set of cuts in the $D(P, T)$.

Notice that $\mathscr{C}_{P, T}$ is not equal to $\mathscr{C}_{\widetilde{P}, T}$ in general, because our construction of $\widetilde{P}$ adds the center cut $n+1$ whenever $p_{i}>t_{i+1}=n+2$. By Proposition 6.16, that is the only change in the cut set, and the addition of the center cut does not alter the equations in these cases.

In fact, by Proposition 6.16, $Z_{P, T}$ and $Z_{\widetilde{P}, T}$ satisfy the same quadratic equations: namely, $\left\{f_{c} \mid c \in\left(\mathscr{C}_{P, T} \cap[1, n]\right) \cup\{n+1\}\right\}$. The following proposition shows that they satisfy the same linear equations as well:

Proposition 6.18. $\mathscr{L}_{P, T}=\mathscr{L}_{\widetilde{P}, T}$.

Proof. Suppose $c \in \mathscr{L}_{\widetilde{P}, T}$. If $c$ is a zero column in $D(\widetilde{P}, T)$, then $\tilde{p}_{i}<c<t_{i+1}$ for some $i$. Whether $\tilde{p}_{i}$ satisfies $(\star)$ or $(\$)$, we then have $c \in \mathscr{L}_{P, T}$.

If $c$ is not a zero column in the diagram, then column $N+1-c$ contains a lone star in $D(\widetilde{P}, T)$. If neither $N+1-c$ nor $N-c$ are exceptional cuts in $D(\widetilde{P}, T)$ then we must have $t_{i}=\tilde{p}_{i}=N+1-c$ for some $i$, by Proposition 2.6. Thus $t_{i} \in \mathscr{C}_{\widetilde{P}, T}$, and hence, by Proposition $6.16, t_{i} \in \mathscr{C}_{P, T}$. It follows that $\left(i, t_{i}\right)$ is a lone star in $D(P, T)$ and $c \in \mathscr{L}_{P, T}$. On the other hand, if either $N+1-c$ or $N-c$ is an exceptional cut in $D(P, T)$ then, by Corollary $6.14, n+1$ must be that exceptional cut. It follows that $c \in\{n+1, n+2\}$. Since $c \in \mathscr{L}_{\widetilde{P}, T}$, we have that $n$ and $n+2$ are in $\mathscr{C}_{\widetilde{P}, T}$, and hence in $\mathscr{C}_{P, T}$ by Proposition 6.16. By Lemma 4.14, either $n+1$ or $n+2$ (that is, either $c$ or $N+1-c$ ) must be in $\mathscr{L}_{P, T}$. But column $N+1-c$ contains a lone star in $D(\widetilde{P}, T)$, and hence $N+1-c \in T \cup \widetilde{P}$. Thus by Corollary 4.13 and Observation 6.10, it is impossible for $N+1-c$ to be in $\mathscr{L}_{P, T}$. Therefore $c \in \mathscr{L}_{P, T}$. It follows that $\mathscr{L}_{\widetilde{P}, T} \subset \mathscr{L}_{P, T}$.

On the other hand, if $c \in \mathscr{L}_{P, T}$, then $c-1$ and $c$ are both cuts in $D(P, T)$. By Proposition 6.16, they are both cuts in $D(\widetilde{P}, T)$ as well, and therefore either $c$ or 
$N+1-c$ is in $\mathscr{L}_{\widetilde{P}, T}$ by Lemma 4.14. If $N+1-c \in \mathscr{L}_{\widetilde{P}, T}$, then we have shown that $N+1-c \in \mathscr{L}_{P, T}$ as well. In that case, by Corollary 4.13, $D(P, T)$ does not have a lone star in column $N+1-c$, so $c$ must be a zero column in $D(P, T)$. In other words $p_{i}<c<t_{i+1}$ for some $i$. But then $\tilde{p}_{i}=p_{i}$, so $c$ is a zero column in $D(\widetilde{P}, T)$ as well.

We have condition (2) of Proposition 6.2 as an immediate corollary:

Corollary 6.19. $Z_{P, T}=Z_{\widetilde{P}, T}$.

Finally, we prove that $\widetilde{P} \preceq P$, and hence that the Richardson variety $Y_{\widetilde{P}, T}$ is indeed contained in $Y_{P, T}$. Our proof will require the following somewhat technical lemma:

Lemma 6.20. Suppose $\mathfrak{t}(T) \neq \mathfrak{t}(P)$, and that there exists an integer $1 \leq c \leq n$ such that $c \notin[T]$ and $[c+1, n+1] \subset[P] \cap[T] \cap[\widetilde{P}]$. Then $c \notin[P] \cap[\widetilde{P}]$.

Proof. Suppose for the sake of contradiction that $c \in[P] \cap[\widetilde{P}]$. We divide our argument into four cases.

Case 1: $c \in \widetilde{P}$ and $N+1-c \in P$. In this case, $\tilde{p}_{i}=c$ for some $i \in[1, m]$ and $p_{j}=N+1-c$ for some $j \in[1, m]$.

Since $c \notin T, \tilde{p}_{i}$ is defined by $(\star)$ or $(\$)$. Hence $\tilde{p}_{i} \in \mathscr{C}_{P, T}$ by Observation 6.11 . Since $p_{j}-1=N-\tilde{p}_{i}$ is also a cut in $D(P, T)$, it follows that $\left(j, p_{j}\right)$ is a lone star in $D(P, T)$, and therefore that $\tilde{p}_{i} \in \mathscr{L}_{P, T}$, contradicting Observation 6.10.

Case 2: $c \in P$ and $N+1-c \in \widetilde{P}$. In this case, $p_{i}=c$ for some $i \in[1, m]$ and $\tilde{p}_{j}=N+1-c$ for some $j \in[1, m]$.

Since $N+1-c \notin T, \tilde{p}_{j}$ is defined by $(\star)$ or $(+)$. Hence $\tilde{p}_{j} \in \mathscr{C}_{P, T}$ by Observation 6.11. Since $p_{i}-1=N-\tilde{p}_{j}$ is also a cut in $D(P, T)$, it follows that $\left(i, p_{i}\right)$ is a lone star in $D(P, T)$, and therefore that $\tilde{p}_{j} \in \mathscr{L}_{P, T}$, contradicting Observation 6.10 .

Case 3: $c \in \widetilde{P} \cap P$. In this case, $p_{i}=c$ for some $i$.

Let $\ell:=n+1-c$. Since $[c+1, n+1] \subset[P]$, Lemma 4.6 tells us that $\left[p_{i+1}, p_{i+\ell}\right] \subset[c+1, N-c]$. We will show that $\tilde{p}_{i+1}<c+1$ and that $\tilde{p}_{i+\ell+1}>$ $N-c$, contradicting the assumption that $[c+1, n+1] \subset \widetilde{P}$ and hence the assumption that $\#([c+1, N-c] \cap \widetilde{P})=\ell$ (by Lemma 4.6), since in this case there can be at most $\ell-1$ elements of $\widetilde{P}$ contained in the interval $[c+1, N-c]$.

We will first show that $\tilde{p}_{i+1}<c+1$. Since $c \in \widetilde{P}$, it must be the case that $\tilde{p}_{j}=c$ for some $j \geq i$. We will show that $j=i+1$.

Note that if $p_{i}=\tilde{p}_{i}<t_{i+1}$ then $\#([1, c] \cap T)=\#([1, c] \cap P)$, and hence $T \nprec P$ (since $\mathfrak{t}(T) \neq \mathfrak{t}(P))$, contradicting the assumption that $T \prec P$.

Also note that if $p_{i}=\tilde{p}_{i}=t_{i+1}$, then $t_{i+1}=c$, contradicting the assumption that $c \notin[T]$. 
Therefore, $\tilde{p}_{j}=c$ for some $j>i$. Furthermore, $\tilde{p}_{j}$ is defined by ( $\left.\$\right)$ since $c<t_{j+1}$, and hence $\tilde{p}_{j} \in \mathscr{C}_{P, T}$.

However, neither $c$ nor $N-c$ is a visible cut in $D(P, T)$, since that would imply $T \nprec P$ by Corollary 4.8. It follows that $c$ is an exceptional cut in $D(P, T)$.

Since $c$ is an exceptional cut, and since $p_{i}=c$, row $i+1$ must be the only row crossing from column $c$ to column $c+1$, by Corollary 4.8. In other words $t_{i+1}<c<p_{i+1}$ and $t_{i+2}>c$. Thus $j=i+1$ and $\tilde{p}_{i+1}=\tilde{p}_{j}=c<c+1$.

It remains to show that $\tilde{p}_{i+\ell+1}>N-c$.

Since $[c+1, n+1] \subset[T]$, and since $t_{i+1}<c<t_{i+2}$, Lemma 4.6 tells us that $\left[t_{i+2}, t_{i+\ell+1}\right] \subset[c+1, N-c]$. Furthermore, row $i+\ell+1$ must cross from column $N-c$ to column $N-c+1$, since $N-c+1 \leq p_{i+\ell+1}$.

In fact, $t_{i+\ell+1}<N-c+1<p_{i+\ell+1}$, since $p_{i+\ell+1} \neq N-c+1$ (due to the fact that $c \in P)$. Therefore $N-c+1$ is not a visible cut in $D(P, T)$. It is not even an apparent cut, since $t_{i}<c=p_{i}$. Finally, $N+1-c$ is not an exceptional cut, since $c \notin[T]$. Thus $N+1-c \notin \mathscr{C}_{P, T}$.

Note that $t_{i+\ell+2}>N-c+1$. Thus, if $\tilde{p}_{i+\ell+1}$ is defined by $(\dagger)$, then $\tilde{p}_{i+\ell+1}>$ $N-c+1$. Furthermore, if $\tilde{p}_{i+\ell+1}$ is defined by $(\star)$ or $(\$)$, then since $N+1-c \notin$ $\mathscr{C}_{P, T}$ and $t_{i+\ell+2}>N-c+1$, we must again have $\tilde{p}_{i+\ell+1}>N-c+1$.

Case 4: $N+1-c \in \widetilde{P} \cap P$. As in the previous case, let $\ell:=n+1-c$. We have $p_{j}=N+1-c$ for some $j$. Since $[c+1, n+1] \subset[P]$, we have $\left[p_{j-\ell}, p_{j-1}\right] \subset$ $[c+1, N-c]$ by Corollary 4.8. We will show that $\tilde{p}_{j}>N-c$ and $\tilde{p}_{j-\ell}<c+1$, contradicting the assumption $[c+1, n+1] \subset[\widetilde{P}]$.

We will first show that $\tilde{p}_{j}>N-c$. Suppose for the sake of contradiction that $\tilde{p}_{k}=N+1-c$ for some $k>j$. Then, since $N+1-c \notin T, \tilde{p}_{k}$ is defined by ( $\star$ ) or $(\$)$, and is therefore a cut in $D(P, T)$ by Observation 6.11. Since $c \notin[T]$, it follows that $\tilde{p}_{k}$ is not an exceptional cut. Since $t_{k} \leq \tilde{p}_{k}=p_{j}<p_{k}$, it follows that $\tilde{p}_{k}$ is not a visible cut either.

But $\tilde{p}_{k}$ is a cut, and hence $c-1$ must be a visible cut in $D(P, T)$. Since $c \notin T$, $c$ must be a zero column in $D(P, T)$. But then $c$ is a visible cut, so by Corollary 4.8 $T \nprec P$, a contradiction. It follows that $k=j$, and hence that $\tilde{p}_{j}=N+1-c>N-c$.

It remains to show that $\tilde{p}_{j-\ell}<c+1$. Since $p_{j}=\tilde{p}_{j}=N+1-c$, and since $N+1-c \notin T$, it must be the case that $\tilde{p}_{j}$ is defined by ( $\star$ ), and therefore that $p_{j}$ is a visible cut in $D(P, T)$. It follows that row $j$ is the only row crossing from column $N-c$ to column $N-c+1$, and hence that $c$ and $N-c$ are exceptional cuts in $D(P, T)$ by Corollary 4.8 .

By Lemma $6.15, c$ is a visible cut in $D(\widetilde{P}, T)$. It follows that $\tilde{p}_{j-\ell} \leq c$, since $t_{j-\ell} \leq c$.

We can now prove that $\widetilde{P}$ satisfies the latter half of condition (1) of Proposition 6.2.

Proposition 6.21. $\widetilde{P} \preceq P$. 
Proof. Note that $\widetilde{P} \leq P$ by construction. Assuming $\widetilde{P}<P$, suppose $\widetilde{P} \nprec P$ for the sake of contradiction. It follows that $\mathfrak{t}(\widetilde{P}) \neq \mathfrak{t}(P)$, and that there exists an integer $c \in[1, n]$ such that $[c+1, n+1] \subset[\widetilde{P}] \cap[P]$ and $\#([1, c] \cap \widetilde{P})=\#([1, c] \cap P)$.

Case 1: $n+1 \notin[T]$. Since $n+1 \in[\widetilde{P}]$, we have $\tilde{p}_{i} \in\{n+1, n+2\}$ for some $i$. Since $n+1 \notin[T], \tilde{p}_{i}$ must be defined by $(\star)$ or $(\$)$. By Observation $6.11, \tilde{p}_{i} \in \mathscr{C}_{P, T}$. If $\tilde{p}_{i}=n+1$, this means $n+1 \in \mathscr{C}_{P, T}$. If $\tilde{p}_{i}=n+2$, then also we have $n+1 \in \mathscr{C}_{P, T}$. To see why, note that both $n$ and $n+1$ are in $2_{P, T}$, so by Lemma 4.14 either $n+1$ or $n+2$ is in $\mathscr{L}_{P, T}$.

If $n+1$ is an exceptional center cut in $D(P, T)$, then $p_{j}=n+2<t_{j+1}$ for some $j$, since $n+1 \notin[T]$. In this case, $\tilde{p}_{j}=p_{j}=n+2$, and hence $n+1$ is a visible cut in $D(P, \widetilde{P})$. On the other hand, if $n+1$ is a visible cut in $D(P, T)$, then it must be a visible cut in $D(P, \widetilde{P})$ as well. It follows that $\#([1, n+1] \cap P)=\#([1, n+1] \cap \widetilde{P})$. In other words $\mathfrak{t}(P)=\mathfrak{t}(\widetilde{P})$, a contradiction.

Case 2: $n+1 \in[T]$. Since $n+1 \in[T] \cap[\widetilde{P}]$, Lemma 6.12 implies $\mathfrak{t}(T)=\mathfrak{t}(\widetilde{P})$. Since we are assuming $\mathfrak{t}(\widetilde{P}) \neq \mathfrak{t}(P)$, this means $\mathfrak{t}(T) \neq \mathfrak{t}(P)$. We can therefore invoke Lemma $6.20 n-c$ times to deduce that $[c+1, n+1] \subset[T]$.

We divide the remainder of the proof into three subcases, depending on the number of rows crossing from column $c$ to column $c+1$ of $D(P, T)$.

Case 2a: $\#([1, c] \cap T)=\#([1, c] \cap P)$. Since $\mathfrak{t}(T) \neq \mathfrak{t}(P)$ and $[c+1, n+1] \subset$ $[T] \cap[P]$, we have $T \not \subset P$, a contradiction.

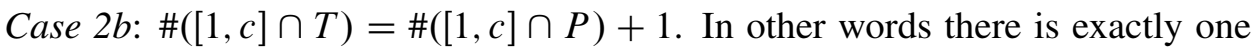
integer $i$ such that $t_{i} \leq c<p_{i}$. Furthermore, $c$ is an exceptional cut in $D(P, T)$, since $[c+1, n+1] \subset[T]$ and $\mathfrak{t}(T) \neq \mathfrak{t}(P)$. Therefore, by Lemma 6.15, $c$ is a visible cut in $D(\widetilde{P}, T)$. This means $\tilde{p}_{i} \leq c<t_{i+1}$. But $c<p_{i}$, so we have $\tilde{p}_{i} \leq c<p_{i}$, contradicting the assumption that $\#([1, c] \cap \widetilde{P})=\#([1, c] \cap P)$.

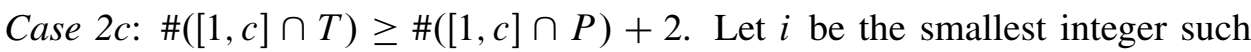
that $t_{i} \leq c<p_{i}$. Note that $t_{i+1} \leq c<p_{i+1}$. Since $\tilde{p}_{i} \leq t_{i+1} \leq c$, it follows that $\#([1, c] \cap \tilde{P})>\#([1, c] \cap P)$, again contradicting the assumption that $\#([1, c] \cap \widetilde{P})=$ $\#([1, c] \cap P)$.

Combining Propositions 6.17 and 6.21, and Corollaries 6.13 and 6.19, we see that the Schubert symbol $\widetilde{P}$ satisfies all three conditions of Proposition 6.2. By the discussion at the beginning of Section 6, this completes the proof of Theorem 1.1.

\section{The Grothendieck ring}

In this section we summarize some facts about $K$-theory which will be used in Sections 8 and 9. Further details can be found in [Fulton 1998] or [Brion 2005].

Given an algebraic variety $X$, let $K^{0}(X)$ denote the Grothendieck ring of algebraic vector bundles on $X$. Let $K_{0}(X)$ denote the Grothendieck group of coherent 
$\mathrm{O}_{X}$-modules, which is a module over $K^{0}(X)$. Both the ring structure of $K^{0}(X)$ and the module structure of $K_{0}(X)$ are defined by tensor products. A closed subvariety $Z \subset X$ has a Grothendieck class $\left[\mathrm{O}_{Z}\right] \in K_{0}(X)$ defined by its structure sheaf. If $X$ is nonsingular, the map $K^{0}(X) \rightarrow K_{0}(X)$ sending a vector bundle to its sheaf of sections is an isomorphism, and we write $K(X):=K_{0}(X) \cong K^{0}(X)$, which we refer to as the Grothendieck ring of $X$.

A morphism of varieties $f: X \rightarrow Y$ defines a pullback ring homomorphism $f^{*}: K^{0}(Y) \rightarrow K^{0}(X)$ by pullback of vector bundles. If $f$ is proper, then there exists a pushforward group homomorphism $f_{*}: K_{0}(X) \rightarrow K_{0}(Y)$. Both these maps are functorial with respect to composition of morphisms. The projection formula says that $f_{*}$ is a $K^{0}$-module homomorphism, in the sense that

$$
f_{*}\left(f^{*} \mathscr{A} \cdot \mathscr{B}\right)=\mathscr{A} \cdot f_{*} \mathscr{B},
$$

where $\mathscr{A} \in K^{0}(Y)$ and $\mathscr{B} \in K_{0}(X)$. If $X$ is a complete variety, then the sheaf Euler characteristic map $\chi_{X}: K(X) \rightarrow K$ (point) $=\mathbb{Z}$ is defined to be the pushforward along the morphism $X \rightarrow$ point.

We need the following well-known fact (see, e.g., [Buch and Ravikumar 2012, Lemma 2.2]).

Lemma 7.1. Let $X$ be a nonsingular variety, and let $Y$ and $Z$ be closed varieties of $X$ with Cohen-Macaulay singularities. Assume that each component of $Y \cap Z$ has dimension $\operatorname{dim}(Y)+\operatorname{dim}(Z)-\operatorname{dim}(X)$. Then $Y \cap Z$ is Cohen-Macaulay and $\left[\mathrm{O}_{Y}\right] \cdot\left[\mathrm{O}_{Z}\right]=\left[\mathrm{O}_{Y \cap Z}\right]$ in $K(X)$.

Finally we recall some facts about the $K$-theory of the projective space $\mathbb{P}^{N-1}$. Let $h \in K\left(\mathbb{P}^{N-1}\right)$ be the class of a hyperplane. Then $h^{j}$ is the class of a codimension- $j$ linear subvariety, $2 h-h^{2}$ is the class of a quadric hypersurface, and $K\left(\mathbb{P}^{N-1}\right)=$ $\mathbb{Z}[h] /\left(h^{N}\right)$. The sheaf Euler characteristic $\chi_{\mathbb{P}^{N-1}}: K\left(\mathbb{P}^{N-1}\right) \rightarrow \mathbb{Z}$ is determined by $h^{j} \mapsto 1$ for $1 \leq j<N$.

\section{Computing triple intersection numbers}

Let $X:=\mathrm{IG}_{\omega}\left(m, \mathbb{C}^{N}\right)$ be a Grassmannian of type $B, C$, or $D$. In this section, we calculate the $K$-theoretic Pieri-type triple intersection numbers

$$
\chi_{X}\left(\left[\mathrm{O}_{X_{P}}\right] \cdot\left[{ }^{\mathrm{O}_{X}}\right] \cdot\left[\mathrm{O}_{X_{(r)}}\right]\right),
$$

where $T \preceq P$ are Schubert symbols in $\Omega(X)$ and $X_{(r)} \subset X$ is a special Schubert variety, which we will define shortly for each type of Grassmannian. Our technique relies on the projection formula to move our calculation to the $K$-theory of projective space. A similar technique was used for the type- $A$ Grassmannian in [Buch and Ravikumar 2012]. 
Recall the projections $\pi$ and $\psi$ from $\operatorname{IF}:=\operatorname{IF}_{\omega}\left(1, m, \mathbb{C}^{N}\right)$ to $X$ and $Z:=$ $\mathrm{IG}_{\omega}\left(1, \mathbb{C}^{N}\right)$ respectively. Since the $K$-theoretic pushforward is functorial with respect to composition, the following diagram commutes:

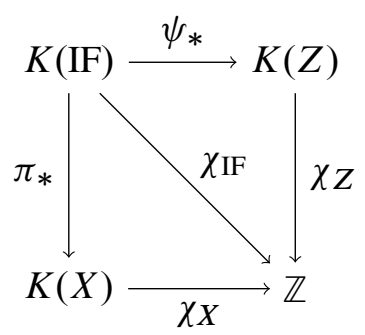

Lemma 8.1. Let $X:=\operatorname{IG}_{\omega}\left(m, \mathbb{C}^{N}\right)$ be a Grassmannian of type $B, C$, or D. Suppose there exists a Schubert variety $W \subset Z:=\operatorname{IG}_{\omega}\left(1, \mathbb{C}^{N}\right)$ such that $\pi\left(\psi^{-1}(W)\right)=$ $X_{(r)}$. We then have

$$
\chi_{X}\left(\left[\mathrm{O}_{X_{P}}\right] \cdot\left[{ }^{O_{X}}\right] \cdot\left[{ }^{\circ} X_{(r)}\right]\right)=\chi_{Z}\left(\left[{ }^{O_{Z}} Z_{P, T}\right] \cdot\left[{ }^{O} W\right]\right)
$$

for any Schubert symbols $T \preceq P$.

Proof. Since $\pi^{-1}\left(X_{P}\right)$ and $\pi^{-1}\left(X^{T}\right)$ are opposite Schubert varieties in IF, it follows that $\pi^{-1}\left(Y_{P, T}\right)$ is a Richardson variety in IF (see, e.g., [Brion 2005]). By [Knutson et al. 2014, Theorem 4.5] or [Billey and Coskun 2012, Theorem 3.3], the projection $\psi: \pi^{-1}\left(Y_{P, T}\right) \rightarrow Z_{P, T}$ is cohomologically trivial, in the sense that $\psi_{*}\left[0_{\pi^{-1}\left(Y_{P, T}\right)}\right]=\left[{ }^{0} Z_{P, T}\right]$. Since $\pi$ is flat, it follows that

$$
\psi_{*} \pi^{*}\left[\mathrm{O}_{Y_{P, T}}\right]=\left[\mathrm{O}_{Z_{P, T}}\right] \in K(Z) .
$$

Similarly, $\pi: \psi^{-1}(W) \rightarrow X_{(r)}$ is cohomologically trivial and $\pi$ is flat, so we have

$$
\pi_{*} \psi^{*}\left[\mathrm{O}_{W}\right]=\left[\mathrm{O}_{X_{(r)}}\right] \in K(X) .
$$

It is known that all Schubert varieties have rational singularities [Mehta and Srinivas 1987]. Therefore by Lemma 7.1 and two applications of the projection formula, we have

$$
\begin{aligned}
& \chi_{X}\left(\left[{ }^{O_{X}} X_{P}\right] \cdot\left[{ }^{O_{X}}\right] \cdot\left[{ }^{O_{X}} X_{(r)}\right]\right)=\chi_{X}\left(\left[{ }^{O_{Y}} Y_{P, T}\right] \cdot \pi_{*} \psi^{*}\left[{ }{ }_{W}\right]\right) \\
& =\chi_{\mathrm{IF}}\left(\pi^{*}\left[\mathrm{O}_{Y_{P, T}}\right] \cdot \psi^{*}\left[\mathrm{O}_{W}\right]\right) \\
& =\chi_{Z}\left(\psi_{*} \pi^{*}\left[\mathrm{O}_{Y_{P, T}}\right] \cdot\left[\mathrm{O}_{W}\right]\right) \\
& =\chi_{Z}\left(\left[\mathrm{O}_{Z_{P, T}}\right] \cdot\left[\mathrm{O}_{W}\right]\right) \text {. }
\end{aligned}
$$

By Lemma 8.1, the projected Richardson variety $Z_{P, T}$ assumes a crucial role in the calculation of (4). Let $q$ be the number of quadratic equations and let $l$ be the number of linear equations defining $Z_{P, T}$ as a complete intersection in $\mathbb{P}^{N-1}$ (from Sections 2 and 4 we know that $q=\#\left\{c \in \mathscr{2}_{P, T}: c>0\right.$ and $\left.c-1 \notin \mathscr{2}_{P, T}\right\}$ 
and $l=\# \mathscr{L}_{P, T}$ ). We now calculate (4) when $X$ has Lie-type $C, B$, or $D$, in that order, finally presenting a unified treatment in Corollary 8.7.

8A. Type $C$. Let $X=\mathrm{SG}(m, 2 n)$. Note that $\mathrm{SG}(1,2 n)$, the image of $\psi$, is equal to $\mathbb{P}^{2 n-1}$. The codimension- $r$ special Schubert variety $X_{(r)}$ is defined for $1 \leq r \leq$ $2 n-m$ by

$$
X_{(r)}:=\left\{\Sigma \in X: \operatorname{dim}\left(\Sigma \cap E_{2 n-m-r+1}\right) \geq 1\right\}
$$

In other words, $X_{(r)}=\pi\left(\psi^{-1}(W)\right)$, where $W:=\mathbb{P}\left(E_{2 n-m-r+1}\right)$. Note that $W$ is a linear subvariety of $\mathbb{P}^{2 n-1}$ and therefore a Schubert variety.

Proposition 8.2. Let $X:=\mathrm{SG}(m, 2 n)$. Given $T \preceq P$ in $\Omega(X)$ and $1 \leq r \leq 2 n-m$, we have

$$
\chi_{X}\left(\left[\mathrm{O}_{X_{P}}\right] \cdot\left[\mathrm{O}_{X^{T}}\right] \cdot\left[\mathrm{O}_{X_{(r)}}\right]\right)=\sum_{j=0}^{2 n-m-r-l-q}\left(\begin{array}{c}
q \\
j
\end{array}\right)(-1)^{j}(2)^{q-j},
$$

where we define $\left(\begin{array}{l}q \\ j\end{array}\right)$ to be zero for $j>q$.

Proof. The triple intersection number is equal to $\chi_{\mathbb{P}^{2 n-1}}\left(\left[\mathrm{O}_{Z_{P, T}}\right] \cdot\left[{ }^{W}\right]\right)$, by Lemma 8.1. Since $Z_{P, T}$ is a complete intersection defined by $l$ linear and $q$ quadratic polynomials, we have $\left[{ }^{0} Z_{P, T}\right]=h^{l} h^{q}(2-h)^{q}$. Since $\left[\mathrm{O}_{W}\right]=h^{m-1+r}$, it follows that

$$
\begin{aligned}
{\left[\mathrm{O}_{\left.Z_{P, T}\right] \cdot\left[\mathrm{O}_{W}\right]}\right.} & =h^{m+r+l+q-1}(2-h)^{q} \\
& =\sum_{j=0}^{2 n-m-r-l-q} h^{m+r+l+q-1}\left(\begin{array}{l}
q \\
j
\end{array}\right)(-h)^{j}(2)^{q-j},
\end{aligned}
$$

where we define $\left(\begin{array}{l}q \\ j\end{array}\right)$ to be zero for $j>q$. Taking sheaf Euler characteristic yields the desired triple intersection formula.

8B. Type B. Let $X:=\mathrm{OG}(m, 2 n+1)$. Let $Q:=\mathrm{OG}(1,2 n+1)$ denote the quadric hypersurface of isotropic lines in $\mathbb{P}^{2 n}$, with inclusion $\iota: Q \hookrightarrow \mathbb{P}^{2 n}$.

We describe the Schubert varieties relative to $E_{\bullet}$ for the odd-dimensional quadric $Q$. For $0 \leq j \leq 2 n-1$ there is exactly one codimension- $j$ Schubert variety $Q_{(j)} \subset Q$, defined by

$$
Q_{(j)}= \begin{cases}\mathbb{P}\left(E_{2 n+1-j}\right) \cap Q & \text { if } 0 \leq j \leq n-1, \\ \mathbb{P}\left(E_{2 n-j}\right) & \text { if } n \leq j \leq 2 n-1 .\end{cases}
$$

The Schubert varieties $Q_{(j)}$ have a straightforward Bruhat ordering:

$$
\begin{aligned}
& \mathbb{P}\left(E_{1}\right) \quad \mathbb{P}\left(E_{2}\right) \quad \mathbb{P}\left(E_{n}\right) \\
& Q_{(2 n-1)} \subset Q_{(2 n-2)} \subset \cdots \subset Q_{(n)} \subset Q_{(n-1)} \subset \cdots \subset Q_{(1)} \subset Q_{(0)}=Q . \\
& \mathbb{P}\left(E_{n+2}\right) \cap Q \quad \mathbb{P}\left(E_{2 n}\right) \cap Q
\end{aligned}
$$


We mention some facts about the Schubert classes in $K(Q)$ (see [Buch and Samuel 2014] for details). For $0 \leq j \leq n-1$, we have $\left[{ }^{0} Q_{(j)}\right]=\iota^{*}\left(h^{j}\right)$. Pushforwards of Schubert classes are given by

$$
\iota_{*}\left[\mathbb{O}_{Q_{(j)}}\right]= \begin{cases}h^{j}\left(2 h-h^{2}\right) & \text { for } 0 \leq j \leq n-1, \\ h^{j+1} & \text { for } n \leq j \leq 2 n-1 .\end{cases}
$$

Returning to the type- $B$ Grassmannian $X$, the codimension- $r$ special Schubert variety $X_{(r)}$ is defined by

$$
X_{(r)}=\left\{\Sigma \in X: \mathbb{P}(\Sigma) \cap Q_{(m+1-r)} \neq \varnothing\right\}
$$

for $1 \leq r \leq 2 n-m$. In other words, $X_{(r)}=\pi\left(\psi^{-1}\left(Q_{(m+1-r)}\right)\right)$.

We now rewrite the type- $B$ triple intersection number as the sheaf Euler characteristic of a $K\left(\mathbb{P}^{2 n}\right)$ class. The final step of computing Euler characteristic is exactly the same as in Proposition 8.2, and is postponed to the unified formula in Corollary 8.7.

Proposition 8.3. Let $X:=\mathrm{OG}(m, 2 n+1)$. For $T \preceq P$ in $\Omega(X)$ and $1 \leq r \leq 2 n-m$, we have

$$
\chi_{X}\left(\left[\mathrm{O}_{X_{P}}\right] \cdot\left[\mathrm{O}_{X^{T}}\right] \cdot\left[\mathrm{O}_{X_{(r)}}\right]\right)
$$

$$
= \begin{cases}\chi_{\mathbb{P}^{2} n}\left(h^{m+r+l+q-1}(2-h)^{q}\right) & \text { if } r \leq n-m, \\ \chi_{\mathbb{P}^{2} n}\left(h^{m+r+l+q-1}(2-h)^{q-1}\right) & \text { if } r>n-m \text { and } q>0, \\ \chi_{\mathbb{P}^{2 n}}\left(h^{m+r+l-1}\right)=0 & \text { if } r>n-m \text { and } q=0 .\end{cases}
$$

Proof. By Lemma 8.1, we must simplify $\chi_{Q}\left(\left[0_{Z_{P, T}}\right] \cdot\left[{ }^{0} Q_{(m-1+r)}\right]\right)$. In certain situations we can use the projection formula along $\iota$ to do this.

Situation 1: Suppose $r \leq n-m$. In this case, $m-1+r \leq n-1$. It follows that the inclusion $\iota\left(Q_{(m-1+r)}\right)$ is a complete intersection in $\mathbb{P}^{2 n}$ cut out by $m-1+r$ linear equations and the single quadratic equation defining $Q$. Thus, $\left[{ }^{0} Q_{(m-1+r)}\right]=$ $\iota^{*}\left(h^{m-1+r}\right)$. Using the projection formula, we have

$$
\begin{aligned}
\chi_{Q}\left(\left[{ }^{Z_{P, T}}\right] \cdot\left[{ }^{0} Q_{(m-1+r)}\right]\right) & =\chi_{Q}\left(\left[\mathbb{O}_{Z_{P, T}}\right] \cdot \iota^{*}\left(h^{m-1+r}\right)\right) \\
& =\chi_{\mathbb{P}^{2 n}}\left(\left[\mathrm{O}_{Z_{P, T}}\right] \cdot h^{m-1+r}\right) \\
& =\chi_{\mathbb{P}^{2 n}}\left(h^{l+q+m+r-1}(2-h)^{q}\right) .
\end{aligned}
$$

Situation 2: Suppose $q$, the number of quadratic equations defining $Z_{P, T}$, is greater than zero. By ignoring one of the quadratic equations defining $Z_{P, T}$, we define a larger subvariety $Z^{\prime} \subset \mathbb{P}^{2 n}$ such that $Z_{P, T}=Z^{\prime} \cap Q$. It follows that 
$\left[{ }^{0} Z_{P, T}\right]=\iota^{*}\left[{ }^{\prime}\right]$, so by the projection formula we have

$$
\begin{aligned}
& \chi_{Q}\left(\left[{ }^{0} Z_{P, T}\right] \cdot\left[{ }^{0} Q_{(m-1+r)}\right]\right) \\
& =\chi_{Q}\left(\iota^{*}\left[{ }^{Z_{Z}}\right] \cdot\left[{ }^{0} Q_{(m-1+r)}\right]\right) \\
& =\chi_{\mathbb{P}^{2 n}}\left(\left[\mathrm{O}_{Z^{\prime}}\right] \cdot \iota_{*}\left[{ }^{\circ} Q_{(m-1+r)}\right]\right) \\
& = \begin{cases}\chi_{\mathbb{P}^{2 n}}\left(h^{l} h^{q-1}(2-h)^{q-1} \cdot h^{m-1+r}\left(2 h-h^{2}\right)\right) & \text { if } r \leq n-m, \\
\chi_{\mathbb{P}^{2 n}}\left(h^{l} h^{q-1}(2-h)^{q-1} \cdot h^{m+r}\right) & \text { if } r>n-m,\end{cases} \\
& = \begin{cases}\chi_{\mathbb{P}^{2 n}}\left(h^{l+q+m+r-1}(2-h)^{q}\right) & \text { if } r \leq n-m, \\
\chi_{\mathbb{P}^{2 n}}\left(h^{l+q+m+r-1}(2-h)^{q-1}\right) & \text { if } r>n-m .\end{cases}
\end{aligned}
$$

Note that these situations are not mutually exclusive, and that when $r \leq n-m$ and $q>0$ both methods agree.

Situation 3: Suppose $r>n-m$ and $q=0$. In this case, both $Z_{P, T}$ and $Q_{(m-1+r)}$ can be thought of as linear subvarieties of $\mathbb{P}^{2 n}$ that are contained in $Q$. Note that $\iota_{*}\left[{ }^{0} Z_{P, T}\right]=h^{l}$ and $\iota_{*}\left[{ }^{0} Q_{(m-1+r)}\right]=h^{m+r}$, but that

$$
\iota_{*}\left(\left[\mathrm{O}_{Z_{P, T}}\right] \cdot\left[\mathrm{O}_{Q_{(m-1+r)}}\right]\right)=h^{m+r+l-1},
$$

since one of these linear equations is redundant in the intersection of generic translates of $Z_{P, T}$ and $Q_{(m-1+r)}$. However, the integer $m+r+l-1$ is at least $2 n+1$, and therefore the Grothendieck class $h^{m+r+l-1} \in K\left(\mathbb{P}^{2 n}\right)$ vanishes.

8C. Type $D$. Let $X:=\mathrm{OG}(m, 2 n+2)$. Let $Q:=\mathrm{OG}(1,2 n+1)$ denote the quadric hypersurface of isotropic lines in $\mathbb{P}^{2 n+1}$ with inclusion $\iota: Q \hookrightarrow \mathbb{P}^{2 n+1}$. We describe the Schubert varieties relative to $E_{\bullet}$ for the even-dimensional quadric $Q$. Let $\widetilde{E}_{n+1}=\left\langle\boldsymbol{e}_{1}, \ldots, \boldsymbol{e}_{n}, \boldsymbol{e}_{n+2}\right\rangle$.

The quadric $Q$ has two Schubert varieties of codimension $n$, defined by

$$
Q_{(n)}:=\mathbb{P}\left(E_{n+1}\right) \quad \text { and } \quad \widetilde{Q}_{(n)}:=\mathbb{P}\left(\widetilde{E}_{n+1}\right) .
$$

For $j \neq n$, there is a single codimension- $j$ Schubert variety, defined by

$$
Q_{(j)}= \begin{cases}\mathbb{P}\left(E_{2 n+2-j}\right) \cap Q & \text { if } 0 \leq j \leq n-1, \\ \mathbb{P}\left(E_{2 n+1-j}\right) & \text { if } n+1 \leq j \leq 2 n .\end{cases}
$$

The Schubert varieties in $Q$ have the following Bruhat order:

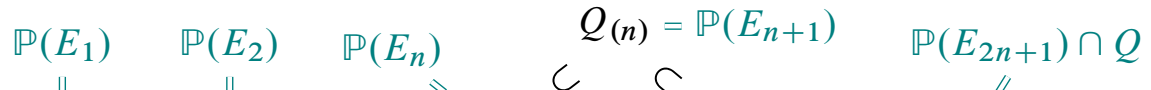

$$
\begin{aligned}
& Q_{(2 n)} \subset Q_{(2 n-1)} \subset \cdots \subset Q_{(n+1)} \quad Q_{(n-1)} \subset \cdots \subset Q_{(1)} \subset Q_{(0)}=Q \\
& \mathbb{P}\left(\widetilde{E}_{n+1}\right)=\widetilde{Q}_{(n)} \quad \mathbb{P}\left(E_{n+3}\right) \cap Q
\end{aligned}
$$


We mention some facts about the Grothendieck ring $K(Q)$, which can be found in [Buch and Samuel 2014, pp. 17-18]. As in type $B$, we have $\left[{ }^{O} Q_{(j)}\right]=\iota^{*}\left(h^{j}\right)$ for $0 \leq j \leq n-1$. Pushforwards of Schubert classes are the same as in type $B$, the only addition being that $\iota_{*}\left[{ }^{0} Q_{(n)}\right]=\iota_{*}\left[{ }^{0} \tilde{Q}_{(n)}\right]=h^{n+1}$. The products of codimension- $n$ classes with one another depend on the parity of $n$, in the sense that

$$
\left[{ }^{0} Q_{(n)}\right]^{2}=\left[{ }^{0} \widetilde{Q}_{(n)}\right]^{2}= \begin{cases}{\left[{ }^{0} Q_{(2 n)}\right]=\left[\mathrm{O}_{\text {point }}\right]} & \text { if } n \text { is even, } \\ 0 & \text { if } n \text { is odd }\end{cases}
$$

whereas

$$
\left[{ }^{0} Q_{(n)}\right] \cdot\left[{ }^{0} \tilde{Q}_{(n)}\right]= \begin{cases}0 & \text { if } n \text { is even, } \\ {\left[{ }^{0} Q_{(2 n)}\right]=\left[{ }^{0} \text { point }\right]} & \text { if } n \text { is odd }\end{cases}
$$

The maximal even orthogonal Grassmannian $\operatorname{OG}(n+1,2 n+2)$ has two connected components. For any $\Sigma \in \mathrm{OG}(n+1,2 n+2)$, let $\mathfrak{t}(\Sigma) \in\{0,1\}$ to be the codimension mod 2 of $\Sigma \cap E_{n+1}$ in $E_{n+1}$. If $L \subset Q$ and $L^{\prime} \subset Q$ are linear subvarieties of codimension $n$ in $Q$, then the affine cones $\Lambda(L)$ and $\Lambda\left(L^{\prime}\right)$ are elements of $\mathrm{OG}(n+1,2 n+2)$. The $K(Q)$ classes $\left[{ }^{\circ}\right]$ and $\left[{ }^{\circ} L^{\prime}\right]$ are equal if and only if $\Lambda(L)$ and $\Lambda\left(L^{\prime}\right)$ are in the same $\mathrm{SO}(2 n+2)$-orbit, which is the case if and only if $\mathfrak{t}(\Lambda(L))=\mathfrak{t}\left(\Lambda\left(L^{\prime}\right)\right)$. For any codimension- $n$ linear subvariety $L \subset Q$, we let $\mathfrak{t}\left(\left[\mathrm{O}_{L}\right]\right):=\mathfrak{t}(\Lambda(L))$. It is easy to check that $\mathfrak{t}\left(\left[{ }^{0} Q_{(n)}\right]\right)=0$ and $\mathfrak{t}\left(\left[{ }^{0} \widetilde{Q}_{(n)}\right]\right)=1$. Hence, for $\mathscr{A}$ and $\mathscr{B}$ in $\left\{\left[{ }^{0} Q_{(n)}\right],\left[{ }^{0} \widetilde{Q}_{(n)}\right]\right\}$, we have

$$
\mathscr{A} \cdot \mathscr{B}=((\mathfrak{t}(\mathscr{A})+\mathfrak{t}(\mathscr{B})+n+1)(\bmod 2))\left[\mathscr{O}_{Q_{(2 n)}}\right],
$$

where mod 2 means "remainder mod 2": the coefficient is 1 if $\mathfrak{t}(\mathscr{A})+\mathfrak{t}(\mathscr{B})+n+1$ is odd, and 0 otherwise.

The following lemma describes $\mathfrak{t}\left(\left[\mathrm{O}_{Z_{P, T}}\right]\right)$ whenever $Z_{P, T}$ has codimension $n$ in $Q$. The lemma is adapted from the definition of the function $h(P, T)$ in [Buch et al. 2009, §5.2].

Lemma 8.4. If $Z_{P, T}$ is a linear subvariety of codimension $n$ in the quadric $Q$, then $\mathfrak{t}\left(Z_{P, T}\right) \equiv|S|+\left|S^{\prime}\right|+n+1(\bmod 2)$, where

$$
\begin{gathered}
S=\left\{i \in[1, n+1]: t_{j} \leq i \leq p_{j} \text { for some } j\right\}, \\
S^{\prime}=\{p \in P: p \geq n+2 \text { and } 2 n+3-p \in S\} .
\end{gathered}
$$

Proof. Let $\Lambda\left(Z_{P, T}\right) \subset \mathbb{C}^{2 n+2}$ be the affine cone over $Z_{P, T} \subset \mathbb{P}^{2 n+1}$. Note that $|S|$ is the number of $c \in[1, n+1]$ such that $c$ is not a zero column in $D(P, T)$, and that $\left|S^{\prime}\right|$ is the number of nonzero columns $c \in[1, n+1]$ such that column $N+1-c$ of $D(P, T)$ contains a lone star. It follows that $|S|-\left|S^{\prime}\right|$ is the number of $c \in[1, n+1]$ such that $\boldsymbol{e}_{c} \in \Lambda\left(Z_{P, T}\right)$, and hence that $n+1-\left(|S|-\left|S^{\prime}\right|\right)$ is the codimension of $\Lambda\left(Z_{P, T}\right) \cap E_{n+1}$ in $E_{n+1}$. 
We give an example in which $\mathfrak{t}\left(\left[\mathrm{O}_{Z_{P, T}}\right]\right.$ ) affects a product in $K(Q)$ (which is in fact a triple intersection number).

Example 8.5. Consider $O G(2,8)$, and let $P=\{1,4\}$ and $T=\{1,2\}$. In this case

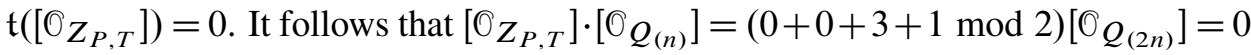
and $\left[{ }^{0} Z_{P, T}\right] \cdot\left[{ }^{0} \tilde{Q}_{(n)}\right]=(0+1+3+1 \bmod 2)\left[{ }^{0} Q_{(2 n)}\right]=\left[{ }^{0} Q_{(2 n)}\right]$.

Returning to the even orthogonal Grassmannian $X$, the codimension- $r$ special Schubert variety $X_{(r)}$ is defined by $X_{(r)}:=\left\{\Sigma \in X: \mathbb{P}(\Sigma) \cap Q_{(m-1+r)} \neq \varnothing\right\}$ for $1 \leq r \leq 2 n+1-m$. As in the quadric case, there is an additional special Schubert variety $^{3}$ of codimension $k:=n-m+1$, defined by $\widetilde{X}_{(k)}:=\left\{\Sigma \in X: \mathbb{P}(\Sigma) \cap \widetilde{Q}_{(n)} \neq \varnothing\right\}$. Thus $X_{(r)}=\pi\left(\psi^{-1}\left(Q_{(m-1+r)}\right)\right)$ for $1 \leq r \leq 2 n+1-m$ and $\tilde{X}_{(k)}=\pi\left(\psi^{-1}\left(\widetilde{Q}_{(n)}\right)\right)$.

Consider the triple intersection number corresponding to $X_{P}, X^{T}$, and a codimension- $r$ special Schubert variety. By Lemma 8.1, this equals $\chi_{Q}\left(\left[{ }^{0} Z_{P, T}\right] \cdot \mathscr{A}\right)$, where $\mathscr{A}$ is the corresponding codimension- $(m-1+r)$ special Schubert class in $K(Q)$. We now translate this expression to the sheaf Euler characteristic of a $K\left(\mathbb{P}^{2 n+1}\right)$ class. The final step of computing Euler characteristic is postponed to the unified formula in Corollary 8.7.

Proposition 8.6. Let $X:=\mathrm{OG}(m, 2 n+2)$, and let $\mathscr{A}$ be a codimension- $(m-1+r)$ Schubert class in $K(Q)$. Define $\delta \in\{0,1\}$ by

$$
\delta \equiv \begin{cases}\mathfrak{t}(\mathscr{A})+|S|+\left|S^{\prime}\right|(\bmod 2) & \text { if } r=k, q=0, \text { and } l=n+1, \\ 1(\bmod 2) & \text { otherwise. }\end{cases}
$$

We then have

$$
\chi_{Q}\left(\left[\mathcal{O}_{Z_{P, T}}\right] \cdot \mathscr{A}\right)= \begin{cases}\chi_{\mathbb{P}^{2 n+1}}\left(h^{m+r+l+q-1}(2-h)^{q}\right) & \text { if } r<k, \\ \chi_{\mathbb{P}^{2 n+1}}\left(h^{m+r+l+q-1}(2-h)^{q-1}\right) & \text { if } r \geq k \text { and } q>0, \\ \chi_{\mathbb{P}^{2 n+1}}\left(\delta \cdot h^{m+r+l-1}\right) & \text { if } r \geq k \text { and } q=0 .\end{cases}
$$

Proof. The proof is exactly as in type $B$, except in the case $r \geq k$ and $q=0$. In this case, $Z_{P, T}$ is a linear subvariety of $Q$ of codimension at least $n$. If $r>k$ or if $Z_{P, T}$ has codimension greater than $n$, then $\left[{ }^{0} Z_{P, T}\right] \cdot \mathscr{A}=0$ (in this case $h^{m+r+l-1} \in K\left(\mathbb{P}^{2 n+1}\right)$ is also zero, since $r>n-m+1$ or $\left.l>n+1\right)$. We can therefore assume $\left[{ }^{0} Z_{P, T}\right]$ and $\mathscr{A}$ are both in $\left\{\left[{ }^{0} Q_{(n)}\right],\left[{ }^{0} \widetilde{Q}_{(n)}\right]\right\}$. By Lemma 8.4, it follows that

$$
\begin{aligned}
\chi_{Q}\left(\left[{ }^{O_{Z, T}}\right] \cdot \mathscr{A}\right) & =\mathfrak{t}\left(\left[{ }_{Z_{P, T}}\right]\right)+\mathfrak{t}(\mathscr{A})+n+1(\bmod 2) \\
& =|S|+\left|S^{\prime}\right|+\mathfrak{t}(\mathscr{A})(\bmod 2) .
\end{aligned}
$$

This number agrees with $\chi_{\mathbb{P}^{2 n+1}}\left(\delta \cdot h^{m+r+l-1}\right)$, since $m+r+l-1=2 n+1$.

${ }^{3}$ We note that our definition of the codimension- $k$ special Schubert varieties differs slightly from the definition in [Buch et al. 2009, §3.2], in which $X_{(k)}$ and $\tilde{X}_{(k)}$ are switched when $n$ is odd. 
8D. A general formula. Propositions 8.2, 8.3, and 8.6 are summarized concisely in the following formulation of the triple intersection number, which holds for isotropic Grassmannians of all types:

Corollary 8.7. Let $X:=\mathrm{IG}_{\omega}(m, N)$ be an isotropic Grassmannian, where $N=2 n$ in type $C, N=2 n+1$ in type $B$, and $N=2 n+2$ in type $D$. Let $k=n-m$ in types $B$ and $C$ and let $k=n-m+1$ in type $D$. Given $1 \leq r \leq n+k$, suppose $\mathscr{A} \in K\left(\mathrm{IG}_{\omega}(1, N)\right)$ is a Schubert class of codimension $(m-1+r)$, so that $\pi_{*} \psi^{*} \mathscr{A} \in K(X)$ is a special Schubert class of codimension $r$. Given $T \preceq P$ in $\Omega(X)$, let $l$ and $q$ be the numbers of linear and quadratic equations defining $Z_{P, T}$ as a complete intersection, and let $S$ and $S^{\prime}$ be the sets defined in Lemma 8.4. Define the integers $l^{\prime}, q^{\prime}, \eta$, and $\delta$ as follows:

$$
\begin{aligned}
q^{\prime} & = \begin{cases}q-1 & \text { if } X \text { is orthogonal and } q>0, \\
q & \text { otherwise, }\end{cases} \\
l^{\prime} & = \begin{cases}l+m+r & \text { if } X \text { is orthogonal, } q>0, \text { and } r \geq k, \\
l+m+r-1 & \text { otherwise, }\end{cases} \\
\eta & = \begin{cases}\mathfrak{t}(\mathscr{A})+|S|+\left|S^{\prime}\right| & \text { if } X \text { is type } D, q=0, \text { and } r=k, \\
1 & \text { otherwise, }\end{cases} \\
\delta & = \begin{cases}0 & \text { if } \eta \text { is even, } \\
1 & \text { if } \eta \text { is odd. }\end{cases}
\end{aligned}
$$

We then have

$$
\begin{aligned}
\chi_{X}\left(\left[\mathcal{O}_{X_{P}}\right] \cdot\left[\mathcal{O}_{X^{T}}\right] \cdot \pi_{*} \psi^{*} \mathscr{A}\right) & =\chi_{\mathbb{P}^{N-1}}\left(\delta h^{l^{\prime}}\left(2 h-h^{2}\right)^{q^{\prime}}\right) \\
& =\delta \cdot \sum_{j=0}^{N-1-l^{\prime}-q^{\prime}}\left(\begin{array}{c}
q^{\prime} \\
j
\end{array}\right)(-1)^{j}(2)^{q^{\prime}-j},
\end{aligned}
$$

where we define $\left(\begin{array}{c}q^{\prime} \\ j\end{array}\right)$ to be zero for $j>q^{\prime}$.

\section{Computing Pieri coefficients}

Let $X$ be an isotropic Grassmannian of type $B, C$, or $D$. Given Schubert symbols $P$ and $Q$ and a special Schubert class $\left[{ }^{O_{(r)}}\right]$, the $K$-theoretic structure constant $\mathcal{N}_{P, r}^{Q}(X)$ is the coefficient of $\left[{ }^{O} X_{Q}\right]$ in the Pieri product $\left[{ }^{O_{X}}\right] \cdot\left[{ }^{O} X_{(r)}\right]$. In this section, we compute $\mathcal{N}_{P, r}^{Q}(X)$ as an alternating sum of triple intersection numbers $\chi_{X}\left(\left[{ }^{O_{X}} X_{P}\right] \cdot\left[{ }^{O_{X}}\right] \cdot\left[{ }^{O_{X}} X_{(r)}\right]\right)$, where $T$ ranges over a certain subset of Schubert symbols (if $X$ is type $D$, the special Schubert class $\left[{ }^{[} \tilde{X}_{(K)}\right]$ can be substituted in order to calculate the additional Pieri coefficients $\left.\widetilde{\mathcal{N}}_{P, r}^{Q}(X)\right)$. 
Given Schubert symbols $Q$ and $P$, the Möbius function $\mu(Q, P)$ is defined by

$$
\mu(Q, P)= \begin{cases}1 & \text { if } Q=P, \\ -\sum_{Q \preceq T \prec P} \mu(Q, T) & \text { if } Q \prec P, \\ 0 & \text { otherwise. }\end{cases}
$$

For each $Q \in \Omega(X)$, we define a class $O_{Q}^{*} \in K(X)$ by

$$
\mathcal{O}_{Q}^{*}:=\sum_{T \in \Omega(X)} \mu(Q, T)\left[\mathscr{O}_{X^{T}}\right] .
$$

Lemma 9.1. The class $O_{Q}^{*}$ is the $K$-theoretic dual to ${ }^{\circ} Q$, in the sense that

$$
\chi_{X}\left(\left[\mathrm{O}_{X_{P}}\right] \cdot \mathrm{O}_{Q}^{*}\right)=\delta_{P, Q} .
$$

Proof. Given Schubert symbols $T \preceq P$, the Richardson variety $Y_{P, T}$ is rational [Deodhar 1985] with rational singularities [Brion 2002]. By [Griffiths and Harris 1978, p. 494] it follows that

$$
\chi_{X}\left(\left[\mathrm{O}_{X_{P}}\right] \cdot\left[\mathrm{O}_{X^{T}}\right]\right)= \begin{cases}1 & \text { if } T \preceq P \\ 0 & \text { otherwise }\end{cases}
$$

If $Q \prec P$, then we have

$$
\begin{aligned}
\chi_{X}\left(\left[\mathcal{O}_{X_{P}}\right] \cdot \mathscr{O}_{Q}^{*}\right) & =\sum_{T \in \Omega(X)} \mu(Q, T) \chi_{X}\left(\left[\mathscr{O}_{X_{P}}\right] \cdot\left[\mathscr{O}_{X^{T}}\right]\right) \\
& =\sum_{T \preceq P} \mu(Q, T) \\
& =\sum_{Q \preceq T \prec P} \mu(Q, T)+\mu(Q, P)=0 .
\end{aligned}
$$

If $Q \not \npreceq P$, then for any $T \succeq Q$ we have $T \not \npreceq P$. Thus, $\left[{ }^{O_{X}}\right] \cdot\left[{ }^{O_{X}}\right]=0$ for every Schubert class $\left[{ }^{O_{X}}\right]$ that has nonzero coefficient in $0_{Q}^{*}$. Finally, if $P=Q$ then $\chi_{X}\left(\left[\mathrm{O}_{X_{Q}}\right] \cdot \mathrm{O}_{Q}^{*}\right)=\chi_{X}\left(\left[\mathrm{O}_{X_{Q}}\right] \cdot\left[\mathrm{O}_{X} Q\right]\right)=1$.

Since $\mu(Q, T)=0$ for $Q \not \npreceq T$ and $\left[{ }^{O_{X}}\right] \cdot\left[{ }^{O_{X}}\right]=0$ for $T \not L P$, we have the following corollary:

Corollary 9.2.

$$
\mathcal{N}_{P, r}^{Q}(X)=\sum_{Q \preceq T \preceq P} \mu(Q, T) \chi_{X}\left(\left[\mathscr{O}_{X_{P}}\right] \cdot\left[\mathscr{O}_{X^{T}}\right] \cdot\left[\mathscr{O}_{X_{(r)}}\right]\right) .
$$

It is known that $\mu(Q, T) \in\left\{0,(-1)^{|Q|-|T|}\right\}$ for any Schubert symbols $Q$ and $T$ [Björner and Brenti 2005, Corollary 2.7.10]. In [Ravikumar 2013, Appendix A] a conjectured criterion is stated for when $\mu(Q, T)$ vanishes. We hope Corollary 9.2 will lead to a Pieri formula for $\mathcal{N}_{P, r}^{Q}(X)$ with manifestly alternating signs, in the sense that $(-1)^{|Q|-|P|-r} \mathcal{N}_{P, r}^{Q}(X)=1$ (see [Brion 2002] for a proof of this fact). 
9A. A global rule. We briefly describe how to determine $K$-theoretic dual classes, and hence Pieri coefficients, without a "local" rule, but rather using the global data of the entire Bruhat order. This method requires us to invert an $L \times L$ matrix, where $L$ is the number of Schubert symbols in $\Omega(X)$, and allows for relatively efficient computation of $\mathcal{N}_{P, r}^{Q}(X)$.

Let $\left\{P_{1}, \ldots, P_{L}\right\}$ be the set of Schubert symbols for $X$. Let $\mathrm{O}_{i}:=\left[\mathrm{O}_{X_{P_{i}}}\right]$ and $\mathcal{O}^{i}:=\left[\mathscr{O}_{X} P_{i}\right]$. The sets $\left\{\mathcal{O}_{1}, \ldots, \mathscr{O}_{L}\right\}$ and $\left\{\mathcal{O}^{1}, \ldots, \mathscr{O}^{L}\right\}$ are both additive bases for $K(X)$.

We will use the following four $L \times L$ matrices:

(1) Let $\boldsymbol{M}:=\left(m_{i j}\right)$ be the intersection matrix for $X$, where

$$
m_{i j}= \begin{cases}1 & \text { if } P_{j} \preceq P_{i} \\ 0 & \text { otherwise }\end{cases}
$$

(2) Let $\boldsymbol{C}_{(r)}:=\left(c_{i j}\right)$ be the Pieri coefficient matrix for $X$, where

$$
\mathrm{O}_{i} \cdot \mathrm{O}_{(r)}=c_{i j} \mathrm{O}_{j}
$$

(3) Let $\boldsymbol{T}_{(r)}:=\left(t_{i j}\right)$ be the triple intersection matrix for $X$, where

$$
t_{i j}=\chi_{X}\left(\mathrm{O}_{i} \cdot \mathcal{O}^{j} \cdot \mathrm{O}_{(r)}\right) .
$$

(4) Let $\boldsymbol{D}:=\boldsymbol{M}^{-1}$ be the matrix of duals on $X$.

Let $\boldsymbol{d}^{j}$ denote the $j$-th column vector of $\boldsymbol{D}$, and let $\mathrm{O}_{\boldsymbol{d}^{j}}:=\sum_{k=1}^{L} d_{k j} \mathrm{O}^{k}$.

Observation 9.3. The element $\mathrm{O}_{\boldsymbol{d}}{ }^{j}$ is dual to $\mathrm{O}_{j}$ in the sense that

$$
\chi_{X}\left(\mathrm{O}_{i} \cdot \mathrm{O}_{\boldsymbol{d}^{j}}\right)=\delta_{i, j}
$$

Proof. $\chi_{X}\left(\mathcal{O}_{i} \cdot \mathscr{O}_{\boldsymbol{d}^{j}}\right)=\boldsymbol{m}_{i} \cdot \boldsymbol{d}^{j}$, where $\boldsymbol{m}_{i}$ is the $i$-th row of $\boldsymbol{M}$.

Observation 9.4. The matrix $\boldsymbol{D}$ transforms triple intersection numbers into Pieri coefficients, via the relation

$$
T_{(r)} \cdot \boldsymbol{D}=C_{(r)}
$$

Proof. $\sum_{k=1}^{L} t_{i k} d_{k j}=\sum_{k=1}^{L} \chi_{X}\left(d_{k j} \mathscr{O}_{i} \cdot \mathcal{O}^{k} \cdot \mathscr{O}_{(r)}\right)=\chi_{X}\left(\mathscr{O}_{i} \cdot \mathscr{O}_{\boldsymbol{d}^{j}} \cdot \mathscr{O}_{(r)}\right)=c_{i j}$.

\section{Acknowledgments}

The results of this paper are part of the author's dissertation [Ravikumar 2013]. The author wishes to sincerely thank Anders Buch for his encouragement and motivation over the years. 


\section{References}

[Billey and Coskun 2012] S. Billey and I. Coskun, "Singularities of generalized Richardson varieties", Comm. Algebra 40:4 (2012), 1466-1495. MR 2912998 Zbl 1274.14059

[Björner and Brenti 2005] A. Björner and F. Brenti, Combinatorics of Coxeter groups, Graduate Texts in Mathematics 231, Springer, New York, 2005. MR 2006d:05001 Zbl 1110.05001

[Borel 1956] A. Borel, “Groupes linéaires algébriques”, Ann. of Math. (2) 64 (1956), 20-82. MR 19,1195h Zbl 0070.26104

[Brion 2002] M. Brion, "Positivity in the Grothendieck group of complex flag varieties", J. Algebra 258:1 (2002), 137-159. MR 2003m:14017 Zbl 1052.14054

[Brion 2005] M. Brion, "Lectures on the geometry of flag varieties", pp. 33-85 in Topics in cohomological studies of algebraic varieties, edited by P. Pragacz, Birkhäuser, Basel, 2005. MR 2006f:14058

[Buch and Ravikumar 2012] A. S. Buch and V. Ravikumar, "Pieri rules for the $K$-theory of cominuscule Grassmannians”, J. Reine Angew. Math. 668 (2012), 109-132. MR 2948873 Zbl 1298.14059

[Buch and Samuel 2014] A. S. Buch and M. J. Samuel, “ $K$-Theory of minuscule varieties”, J. Reine Angew. Math. (online publication July 2014).

[Buch et al. 2009] A. S. Buch, A. Kresch, and H. Tamvakis, "Quantum Pieri rules for isotropic Grassmannians”, Invent. Math. 178:2 (2009), 345-405. MR 2011a:14112 Zbl 1193.14071

[Buch et al. 2013] A. S. Buch, A. Kresch, and H. Tamvakis, "A Giambelli formula for even orthogonal Grassmannians", J. Reine Angew. Math. (online publication September 2013).

[Deodhar 1985] V. V. Deodhar, "On some geometric aspects of Bruhat orderings, I: A finer decomposition of Bruhat cells", Invent. Math. 79:3 (1985), 499-511. MR 86f:20045 Zbl 0563.14023

[Fulton 1998] W. Fulton, Intersection theory, 2nd ed., Ergebnisse der Mathematik und ihrer Grenzgebiete (3) 2, Springer, Berlin, 1998. MR 99d:14003 Zbl 0885.14002

[Fulton and Harris 1991] W. Fulton and J. Harris, Representation theory, Graduate Texts in Mathematics 129, Springer, New York, 1991. MR 93a:20069 Zbl 0744.22001

[Griffiths and Harris 1978] P. Griffiths and J. Harris, Principles of algebraic geometry, Wiley, New York, 1978. Reprinted 1994. MR 95d:14001 Zbl 0836.14001

[He and Lam 2011] X. He and T. Lam, "Projected Richardson varieties and affine Schubert varieties", preprint, 2011. arXiv 1106.2586v1

[Kleiman 1974] S. L. Kleiman, "The transversality of a general translate”, Compositio Math. 28 (1974), 287-297. MR 50 \#13063 Zbl 0288.14014

[Knutson et al. 2014] A. Knutson, T. Lam, and D. E. Speyer, "Projections of Richardson varieties", J. Reine Angew. Math. 687 (2014), 133-157. MR 3176610 Zbl 06296354

[Mehta and Srinivas 1987] V. B. Mehta and V. Srinivas, "Normality of Schubert varieties", Amer. J. Math. 109:5 (1987), 987-989. MR 88j:14063 Zbl 0651.14030

[Ravikumar 2013] V. Ravikumar, Triple Intersection Formulas for Isotropic Grassmannians, Ph.D. thesis, Rutgers University, 2013, http://rucore.libraries.rutgers.edu/rutgers-lib/41905/.

[Richardson 1992] R. W. Richardson, "Intersections of double cosets in algebraic groups", Indag. Math. (N.S.) 3:1 (1992), 69-77. MR 93b:20081 Zbl 0833.22001

[Springer 2009] T. A. Springer, Linear algebraic groups, 2nd ed., Birkhäuser, Boston, 2009. MR 2009i:20089 Zbl 1202.20048

Communicated by Ravi Vakil

Received 2014-06-25 Revised 2014-08-07 Accepted 2015-03-07 
vijayr@cmi.ac.in

Mathematics Department, Chennai Mathematical Institute, H1, SIPCOT IT Park, Siruseri, Kelambakkam 603103, India 


\section{Algebra \& Number Theory}

msp.org/ant

\section{EDITORS}

MANAGING EDITOR

Bjorn Poonen

Massachusetts Institute of Technology

Cambridge, USA

\author{
EDITORIAL BOARD CHAIR \\ David Eisenbud \\ University of California \\ Berkeley, USA
}

\section{BOARD OF EDITORS}

Georgia Benkart

Dave Benson

Richard E. Borcherds

John H. Coates

J-L. Colliot-Thélène

Brian D. Conrad

Hélène Esnault

Hubert Flenner

Edward Frenkel

Andrew Granville

Joseph Gubeladze

Roger Heath-Brown

Craig Huneke

János Kollár

Yuri Manin

Barry Mazur

Philippe Michel
University of Wisconsin, Madison, USA

University of Aberdeen, Scotland

University of California, Berkeley, USA

University of Cambridge, UK

CNRS, Université Paris-Sud, France

University of Michigan, USA

Freie Universität Berlin, Germany

Ruhr-Universität, Germany

University of California, Berkeley, USA

Université de Montréal, Canada

San Francisco State University, USA

Oxford University, UK

University of Virginia, USA

Princeton University, USA

Northwestern University, USA

Harvard University, USA

École Polytechnique Fédérale de Lausanne
Susan Montgomery

Shigefumi Mori

Raman Parimala

Jonathan Pila

Anand Pillay

Victor Reiner

Peter Sarnak

Joseph H. Silverman

Michael Singer

Vasudevan Srinivas

J. Toby Stafford

Ravi Vakil

Michel van den Bergh

Marie-France Vignéras

Kei-Ichi Watanabe

Efim Zelmanov

Shou-Wu Zhang
University of Southern California, USA

RIMS, Kyoto University, Japan

Emory University, USA

University of Oxford, UK

University of Notre Dame, USA

University of Minnesota, USA

Princeton University, USA

Brown University, USA

North Carolina State University, USA

Tata Inst. of Fund. Research, India

University of Michigan, USA

Stanford University, USA

Hasselt University, Belgium

Université Paris VII, France

Nihon University, Japan

University of California, San Diego, USA

Princeton University, USA

\section{PRODUCTION}

production@msp.org

Silvio Levy, Scientific Editor

See inside back cover or msp.org/ant for submission instructions.

The subscription price for 2015 is US $\$ 255 /$ year for the electronic version, and $\$ 440 /$ year ( $+\$ 55$, if shipping outside the US) for print and electronic. Subscriptions, requests for back issues and changes of subscribers address should be sent to MSP.

Algebra \& Number Theory (ISSN 1944-7833 electronic, 1937-0652 printed) at Mathematical Sciences Publishers, 798 Evans Hall \#3840, c/o University of California, Berkeley, CA 94720-3840 is published continuously online. Periodical rate postage paid at Berkeley, CA 94704, and additional mailing offices.

ANT peer review and production are managed by EditFLOW ${ }^{\circledR}$ from MSP.

\section{PUBLISHED BY}

- mathematical sciences publishers

nonprofit scientific publishing

http://msp.org/

(C) 2015 Mathematical Sciences Publishers 


\section{Algebra \& Number Theory}

Volume $9 \quad$ No. $3 \quad 2015$

Hurwitz monodromy and full number fields

DAVID P. ROBERTS and AKSHAY VENKATESH

The characteristic polynomial of the Adams operators on graded connected Hopf algebras

Marcelo Aguiar and Aaron Lauve

Secant spaces and syzygies of special line bundles on curves

MARIAN APRODU and EDOARDO SERNESI

Complex group algebras of the double covers of the symmetric and alternating groups

601

Christine Bessenrodt, Hung Ngoc Nguyen, Jørn B. Olsson and Hung P. TONG-VIET

Fano schemes of determinants and permanents

Melody Chan and NATHan Ilten

Triple intersection formulas for isotropic Grassmannians

VIJAY RAVIKUMAR

On the basepoint-free theorem for log canonical threefolds over the algebraic closure of a finite field

Diletta Martinelli, Yusuke NaKamura and Jakub Witaszek

The torsion group of endotrivial modules

JON F. CARLSON and JACQUES THÉVENAZ 